\title{
STABILIZATION OF SECOND ORDER EVOLUTION EQUATIONS BY A CLASS OF UNBOUNDED FEEDBACKS
}

\author{
KAIS Ammari ${ }^{1}$ AND MARIUs TUCSNAK ${ }^{1}$
}

\begin{abstract}
In this paper we consider second order evolution equations with unbounded feedbacks. Under a regularity assumption we show that observability properties for the undamped problem imply decay estimates for the damped problem. We consider both uniform and non uniform decay properties.
\end{abstract}

Mathematics Subject Classification. 93B52, 93D15, 93B07.

Received October 19, 2000. Revised February 19, 2001.

\section{INTRODUCTION}

In recent years an important literature was devoted to the controllability and stabilizability of second order infinite dimensional systems comming from elasticity (see for instance Lions [16] and references therein). According to the classical principle of Russell (see [23]) if a system is uniformly stabilizable by using colocated actuators and sensors then it is exactly controllable by using the same actuators (i.e., the same input operator). As far as we know the converse of this assertion was not proved in a general framework. The only result available in the literature supposes that the input operator is bounded (see Haraux [9]) in the energy space or they are based on non local feed-backs (see for instance Komornik [12] and references therein). In the applications for PDE's systems this situation leads to non-local feedbacks given, in particular, by Riccati type operators. However in many PDE systems the exponential stabilizability with colocated actuators and sensors was proved by direct methods (see Lagnese [14], Komornik and Zuazua [13]) by using multiplier techniques. The aim of this paper is to give a class of unbounded input operators for which exact controllability implies uniform stabilizability by colocated actuators and sensors.

More precisely, let $X$ be a complex Hilbert space with norm and inner product denoted respectively by $\|\cdot\|_{X}$ and $\langle., .\rangle_{X}$. Let $A$ be a linear unbounded self-adjoint and strictly positive operator in $X$. Let $\mathcal{D}\left(A^{\frac{1}{2}}\right)$ be the domain of $A^{\frac{1}{2}}$. Denote by $\left(\mathcal{D}\left(A^{\frac{1}{2}}\right)\right)^{\prime}$ the dual space of $\mathcal{D}\left(A^{\frac{1}{2}}\right)$ obtained by means of the inner product in $X$.

Further, let $U$ be a complex Hilbert space (which will be identified to its dual space) with norm and inner product respectively denoted by $\|.\|_{U}$ and $\langle., .\rangle_{U}$ and let $B \in \mathcal{L}\left(U,\left(\mathcal{D}\left(A^{\frac{1}{2}}\right)\right)^{\prime}\right)$.

Most of the linear control problems comming from elasticity can be written as

$$
\left\{\begin{array}{l}
x^{\prime \prime}(t)+A x(t)+B u(t)=0 \\
x(0)=z^{0}, x^{\prime}(0)=z^{1}
\end{array}\right.
$$

Keywords and phrases: Stabilization, observability inequality, second order evolution equations, unbounded feedbacks.

1 Institut Elie Cartan, Département de Mathématiques, Université de Nancy I, 54506 Vandœuvre-lès-Nancy Cedex, France; e-mail: ammari@iecn.u-nancy.fr; tucsnak@iecn.u-nancy.fr 
where $x:[0, T] \rightarrow X$ is the state of the system, $u \in L^{2}(0, T ; U)$ is the input function and we denoted the differentiation with respect to time by " $"$.

We define the energy of $x(t)$ at instant $t$ by

$$
E(x(t))=\frac{1}{2}\left\{\left\|x^{\prime}(t)\right\|_{X}^{2}+\left\|A^{\frac{1}{2}} x(t)\right\|_{X}^{2}\right\}
$$

Simple formal calculations give

$$
E(x(0))-E(x(t))=-\left\langle B u(t), x^{\prime}(t)\right\rangle_{\mathcal{D}\left(A^{\frac{1}{2}}\right),\left(\mathcal{D}\left(A^{\frac{1}{2}}\right)\right)^{\prime}}, \quad \forall t \geq 0 .
$$

This is why, in many problems, coming in particular from elasticity, the input $u$ is given in the feedback form $u(t)=B^{*} x^{\prime}(t)$, which obviously gives a nonincreasing energy and which corresponds to colocated actuators and sensors. The aim of this paper is to give sufficient conditions making the corresponding closed loop system

$$
\begin{gathered}
x^{\prime \prime}(t)+A x(t)+B B^{*} x^{\prime}(t)=0, \\
x(0)=x^{0}, x^{\prime}(0)=x^{1},
\end{gathered}
$$

uniformly stable in the energy space $\mathcal{D}\left(A^{\frac{1}{2}}\right) \times X$. In the case of non uniform stability we give sufficient conditions for weaker decay properties.

In order to obtain the characterization of decay properties of the damped problem via observability inequalities for the conservative problem we will use the assumption below. This assumption is less restrictive than the boundedness of $B$ which was the basic hypothesis in [9].

(H)

If $\beta>0$ is fixed and $C_{\beta}=\{\lambda \in \mathbb{C} \mid \operatorname{Re} \lambda=\beta\}$, the function

$$
\lambda \in \mathbb{C}_{+}=\{\lambda \in \mathbb{C} \mid \operatorname{Re} \lambda>0\} \rightarrow H(\lambda)=\lambda B^{*}\left(\lambda^{2} I+A\right)^{-1} B \in \mathcal{L}(U)
$$

is bounded on $C_{\beta}$.

An equivalent statement of $(\mathrm{H})$ is given at Section 3. Under this alternative form this assumption can be verified for PDE systems (such as the systems in the examples below), by proving results called (in the PDE community) "hidden regularity results".

The main novelties brought in by this paper are the following:

(a) we give a sufficient and necessary condition for the exponential stability of all finite energy solutions of $(1.3,1.4)$ by using only the undamped problem (i.e. corresponding to $B=0$ in $(1.3)$ );

(b) in the case of non exponential stability in the energy space we give an explicit decay rate for all initial data lying in a more regular space.

Our approach has common points with the result obtained in [9] for feedbacks which are bounded in the energy space. The main difference is that we replace the assumption of boundedness of $B$ by the assumption $(\mathrm{H})$. Moreover our methods are related to those proposed in [28] for a general class of first order systems (see the comming paper [26] for a description of the connections between our results and those in [28]).

The paper is organized as follows. In the second section we give precise statements of the main results. Some regularity results implied by $(\mathrm{H})$ are given in Section 3. Section 4 contains the proof of the main results. The last section is devoted to some applications. 


\section{Statement of the main Results}

Let $x(t)$ be a solution of $(1.3,1.4)$. Simple formal calculations show that a sufficiently smooth solution of $(1.3,1.4)$ satisfies the energy estimate

$$
E(x(0))-E(x(t))=\int_{0}^{t}\left\|B^{*} x^{\prime}(s)\right\|_{U}^{2} \mathrm{~d} s, \quad \forall t \geq 0 .
$$

In particular (2.1) implies that

$$
E(x(t)) \leq E(x(0)), \forall t \geq 0 .
$$

Estimate above suggests that the natural well-posedness space for $(1.3,1.4)$ is $V \times X$ where $V=\mathcal{D}\left(A^{\frac{1}{2}}\right)$ and $\|x\|_{V}=\left\|A^{\frac{1}{2}} x\right\|_{X}, \forall x \in V$.

The existence and uniqueness of finite energy solutions of $(1.3,1.4)$ can be obtained by standard semigroup methods. This why the results below are given without proofs.

Proposition 2.1. Suppose that $\left(x^{0}, x^{1}\right) \in V \times X$. Then the problem $(1.3,1.4)$ admits a unique solution

$$
x(t) \in C(0, T ; V) \cap C^{1}(0, T ; X)
$$

such that $B^{*} x(.) \in H^{1}(0, T ; U)$ and

$$
\left\|\left(B^{*} x\right)^{\prime}(\cdot)\right\|_{L^{2}(0, T ; U)}^{2} \leq C\left\|\left(x^{0}, x^{1}\right)\right\|_{V \times X}^{2},
$$

where the constant $C>0$ is independent of $\left(x^{0}, x^{1}\right)$. Moreover $x(t)$ satisfies the energy estimate $(2.1)$.

Let us now consider the problem

$$
\begin{gathered}
\phi^{\prime \prime}(t)+A \phi(t)=0, \\
\phi(0)=x^{0}, \phi^{\prime}(0)=x^{1} .
\end{gathered}
$$

It is well known that $(2.3,2.4)$ is well-posed in $\mathcal{D}(A) \times V$ and in $V \times X$.

The following theorem is a direct generalisation of the result in [9].

Theorem 2.2. Assume that the hypothesis (H) is verified. Then, the system described by (1.3, 1.4) is exponentially stable in $V \times X$ if and only if there exists $T>0, C>0$ such that

$$
\int_{0}^{T}\left\|\left(B^{*} \phi\right)^{\prime}(t)\right\|_{U}^{2} \mathrm{~d} t \geq C\left\|\left(x^{0}, x^{1}\right)\right\|_{V \times X}^{2}, \forall\left(x^{0}, x^{1}\right) \in \mathcal{D}(A) \times V .
$$

Remark 2.3. Assumption $(\mathrm{H})$ is not necessary for the implication: uniform exponential stability $\Rightarrow(2.5)$. The latter follows from (indirectly) Russell's principle [23].

The statement of our second main result requires some notations.

Consider the unbounded linear operator

$$
\mathcal{A}_{d}: \mathcal{D}\left(\mathcal{A}_{d}\right) \rightarrow V \times X, \mathcal{A}_{d}=\left(\begin{array}{cc}
0 & I \\
-A & -B B^{*}
\end{array}\right),
$$

where

$$
\mathcal{D}\left(\mathcal{A}_{d}\right)=\left\{(u, v) \in V \times X, A u+B B^{*} v \in X, v \in V\right\} .
$$


Let $X_{1}, X_{2}, Y_{1}, Y_{2}$ be four Banach spaces such that

$$
\begin{gathered}
V \times X \subset X_{1} \times X_{2}, \mathcal{D}\left(\mathcal{A}_{d}\right) \subset Y_{1} \times Y_{2} \subset V \times X, \\
\forall z \in \mathcal{D}\left(\mathcal{A}_{d}\right),\|z\|_{\mathcal{D}\left(\mathcal{A}_{d}\right)} \sim\|z\|_{Y_{1} \times Y_{2}}
\end{gathered}
$$

and

$$
\left[Y_{1} \times Y_{2}, X_{1} \times X_{2}\right]_{\theta}=V \times X,
$$

for a fixed real number $\theta \in] 0,1[\text {, where [.,. }]_{\theta}$ denotes the interpolation space (see for instance Triebel [24]).

Let $\mathcal{G}: \mathbb{R}_{+} \rightarrow \mathbb{R}_{+}$such that $\mathcal{G}$ is continuous, invertible, increasing on $\mathbb{R}_{+}$and suppose that the function $x \rightarrow \frac{1}{x^{\frac{\theta}{1-\theta}}} \mathcal{G}(x)$ increasing on $(0,1)$.

In the case of non exponential decay in the energy space we give explicit decay estimates valid for regular initial data, as stated in the result below:

Theorem 2.4. Assume that assumption $(H)$ is verified and that the function $\mathcal{G}$ satisfies assumptions above. Then the following assertions hold true:

1. If for all $\left(x^{0}, x^{1}\right) \in \mathcal{D}(A) \times V$ we have

$$
\int_{0}^{T}\left\|\left(B^{*} \phi\right)^{\prime}(t)\right\|_{U}^{2} \mathrm{~d} t \geq C\left\|\left(x^{0}, x^{1}\right)\right\|_{V \times X}^{2} \mathcal{G}\left(\frac{\left\|\left(x^{0}, x^{1}\right)\right\|_{X_{1} \times X_{2}}^{2}}{\left\|\left(x^{0}, x^{1}\right)\right\|_{V \times X}^{2}}\right),
$$

for some constant $C>0$ then there exists a constant $C_{1}>0$ such that for all $t>0$ and for all $\left(x^{0}, x^{1}\right)$ $\in \mathcal{D}\left(\mathcal{A}_{d}\right)$ we have

$$
E(x(t)) \leq C_{1}\left[\mathcal{G}^{-1}\left(\frac{1}{1+t}\right)\right]^{\frac{\theta}{1-\theta}}\left\|\left(x^{0}, x^{1}\right)\right\|_{\mathcal{D}\left(\mathcal{A}_{d}\right)}^{2} .
$$

2. If for all $\left(x^{0}, x^{1}\right) \in \mathcal{D}(A) \times V$ we have

$$
\int_{0}^{T}\left\|\left(B^{*} \phi\right)^{\prime}(t)\right\|_{U}^{2} \mathrm{~d} t \geq C\left\|\left(x^{0}, x^{1}\right)\right\|_{X_{1} \times X_{2}}^{2},
$$

for some constant $C>0$ then there exists a constant $C_{2}>0$ such that for all $t>0$ and for all $\left(x^{0}, x^{1}\right)$ $\in \mathcal{D}\left(\mathcal{A}_{d}\right)$ we have

$$
E(x(t)) \leq \frac{C_{2}}{(1+t)^{\frac{\theta}{1-\theta}}}\left\|\left(x^{0}, x^{1}\right)\right\|_{\mathcal{D}\left(\mathcal{A}_{d}\right)}^{2} .
$$

Remark 2.5. 1. Estimates similar to (2.11) were first given by Russell [22] in the case of bounded feedback controls. Russell's method cannot be directly extended to unbounded feedbacks.

2. If $\theta \in\left(0, \frac{1}{2}\right)$ then the identity function satisfies the assumptions on $\mathcal{G}$ in Theorem 2.4. In this case (2.11) is a consequence of $(2.9)$. However if $\theta \in\left(\frac{1}{2}, 1\right)$ then the identity function does not satisfy the assumptions on $\mathcal{G}$ in the Theorem 2.4. In this second case (2.11) is not a consequence of (2.9). 


\section{Some Regularity Results}

Consider the evolution problem

$$
\begin{gathered}
y^{\prime \prime}(t)+A y(t)=B v(t), \\
y(0)=y^{\prime}(0)=0 .
\end{gathered}
$$

A natural question is the regularity of $y$ when $v \in L^{2}(0, T ; U)$. By applying standard energy estimates we can easily check that $y \in C(0, T ; X) \cap C^{1}\left(0, T ; V^{\prime}\right)$. However if $B$ satisfies a certain admissibility condition then $y$ is more regular. More precisley the following result, which is a version of the general transposition method (see, for instance, Lions and Magenes [17]), holds true.

Lemma 3.1. Suppose that $v \in L^{2}(0, T ; U)$ and that the solutions $\phi$ of (2.3, 2.4) are such that $B^{*} \phi($. $\in H^{1}(0, T ; U)$ and there exists a constant $C>0$ such that

$$
\left\|\left(B^{*} \phi\right)^{\prime}(\cdot)\right\|_{L^{2}(0, T ; U)} \leq C\left\|\left(x^{0}, x^{1}\right)\right\|_{V \times X}, \quad \forall\left(x^{0}, x^{1}\right) \in V \times X .
$$

Then the problem (3.1, 3.2) admits a unique solution having the regularity

$$
y \in C(0, T ; V) \cap C^{1}(0, T ; X) .
$$

Proof. Let

$$
\mathcal{D}(\mathcal{A})=\mathcal{D}(A) \times V
$$

and denote by $[\mathcal{D}(\mathcal{A})]^{\prime}$ the dual space of $\mathcal{D}(\mathcal{A})$ with respect to the pivot space $V \times X$.

If we put $Z=\left(\begin{array}{c}y \\ y^{\prime}\end{array}\right)$ it is clear that $(3.1,3.2)$ can be written as

$$
Z^{\prime}+\mathcal{A} Z(t)=\mathcal{B} v(t), Z(0)=0
$$

where

$$
\begin{aligned}
& \mathcal{A}=\left(\begin{array}{cc}
0 & -I \\
A & 0
\end{array}\right): V \times X \rightarrow[\mathcal{D}(\mathcal{A})]^{\prime}, \\
& \mathcal{B}=\left(\begin{array}{c}
0 \\
B
\end{array}\right): U \rightarrow[\mathcal{D}(\mathcal{A})]^{\prime} .
\end{aligned}
$$

It well known that $\mathcal{A}$ is a skew adjoint operator so it generates a group of isometries in $[\mathcal{D}(\mathcal{A})]^{\prime}, \operatorname{denoted}$ by $\mathcal{S}(t)$.

After simple calculations we get that the operator $\mathcal{B}^{*}: \mathcal{D}(\mathcal{A}) \rightarrow U$ is given by

$$
\mathcal{B}^{*}\left(\begin{array}{l}
u \\
v
\end{array}\right)=B^{*} v, \forall(u, v) \in \mathcal{D}(\mathcal{A})
$$

This implies that

$$
\mathcal{B}^{*} \mathcal{S}^{*}(t)\left(\begin{array}{l}
x^{0} \\
x^{1}
\end{array}\right)=B^{*} \phi^{\prime}, \forall\left(x^{0}, x^{1}\right) \in \mathcal{D}(\mathcal{A})
$$


with $\phi$ satisfying $(2.3,2.4)$. From the inequality above and (3.3) we deduce that there exists a constant $C>0$ such that

$$
\int_{0}^{T}\left\|\mathcal{B}^{*} \mathcal{S}^{*}(t)\left(\begin{array}{c}
x^{0} \\
x^{1}
\end{array}\right)\right\|_{U}^{2} \mathrm{~d} t \leq C\left\|\left(x^{0}, x^{1}\right)\right\|_{V \times X}^{2}, \forall\left(x^{0}, x^{1}\right) \in \mathcal{D}(\mathcal{A})
$$

According to Theorem 3.1 in [6] (p. 187) the inequality above implies the interior regularity (3.5).

Proposition 3.2. Suppose that $v \in L^{2}(0, T ; U)$ and that the problem (3.1, 3.2) admits a unique solution having the regularity

$$
y \in C(0, T ; V) \cap C^{1}(0, T ; X) .
$$

Then hypothesis (H) holds if and only if $B^{*} y(.) \in H^{1}(0, T ; U)$ and there exists a constant $C>0$ such that

$$
\left\|\left(B^{*} y\right)^{\prime}(\cdot)\right\|_{L^{2}(0, T ; U)} \leq C\|v\|_{L^{2}(0, T ; U)}, \quad \forall v \in L^{2}(0, T ; U) .
$$

Proof. As equation (3.1) is time reversible, after extending $v$ by zero for $t \in \mathbb{R} \backslash[0, T]$, we can solve $(3.1,3.2)$, for $t \in \mathbb{R}$. By this way, we obtain a function, denoted also by $y$, such that

$$
\begin{aligned}
& y \in C(\mathbb{R} ; V) \cap C^{1}(\mathbb{R} ; X) \cap L^{2}(\mathbb{R} ; V), \\
& y(t)=0, \quad \forall t \leq 0,
\end{aligned}
$$

and $y$ satisfies $(3.1,3.2)$ for all $t \in \mathbb{R}$.

Let $\widehat{y}(\lambda)$, where $\lambda=\gamma+i \eta, \gamma>0$ and $\eta \in \mathbb{R}$, be the Laplace (with respect to $t$ ) transform of $y$. Since $y$ satisfies (3.7), estimate (3.6) is equivalent to the fact that the function $t \rightarrow \mathrm{e}^{-\gamma t} B^{*} y(t)$ belongs to $H^{1}(\mathbb{R} ; U)$ and that there exists a constant $M_{1}>0$ such that

$$
\| \mathrm{e}^{-\gamma \cdot B^{*} y(\cdot)\left\|_{H^{1}(\mathbb{R} ; U)}^{2} \leq M_{1}\right\| v(\cdot) \|_{L^{2}(\mathbb{R} ; U)}^{2} .}
$$

Equivalently, by the Parseval identity (see for instance Doetsch [8], p. 212), it suffices to prove that the function

$$
\eta \rightarrow(\gamma+i \eta) B^{*} \widehat{y}(\gamma+i \eta)
$$

belongs to $L^{2}\left(\mathbb{R}_{\eta} ; U\right)$, for some $\gamma>0$, and that there exists a constant $M_{2}>0$ such that

$$
\left\|(\gamma+i \eta) B^{*} \widehat{y}(\gamma+i \eta)\right\|_{L^{2}\left(\mathbb{R}_{\eta} ; U\right)}^{2} \leq M_{2} \int_{-\infty}^{+\infty}\|\widehat{v}(\gamma+i \eta)\|_{U}^{2} \mathrm{~d} \eta .
$$

It can be easily checked that $\widehat{y}$ satisfies:

$$
\lambda^{2} \widehat{y}(\lambda)+A \widehat{y}(\lambda)=B \widehat{v}(\lambda), \forall \operatorname{Re} \lambda>0 .
$$

Relation above implies, for $\operatorname{Re} \lambda>0$ that

$$
\lambda B^{*} \widehat{y}(\lambda)=H(\lambda) \widehat{v}(\lambda), \forall \operatorname{Re} \lambda>0,
$$

where $H(\lambda)$ is defined in (1.5). Assumption (H) implies the existence of a constant $M_{2}>0$ such that (3.8) holds true. This ends the proof of the fact that assumption $(\mathrm{H})$ implies that (3.6) holds for all finite energy solution of $(3.1,3.2)$. 
Suppose now that (3.6) holds true. By using the time reversibility and the invariance with respect to translations (in time) of $(3.1)$ we obtain that $(3.1,3.2)$ is well posed for all input $v \in L^{2}(\mathbb{R}, U)$, $v$ compactly supported. More precisely, we have

$$
\int_{\operatorname{supp}(v)}\left\|\left(B^{*} y\right)^{\prime}(t)\right\|_{U}^{2} \mathrm{~d} t \leq C \int_{\operatorname{supp}(v)}\|v(t)\|_{U}^{2} \mathrm{~d} t,
$$

for all compactly supported $v \in L^{2}(\mathbb{R}, U)$, with the same constant as in (3.6).

Using (3.10) it follows that

$$
\|H(\gamma+i \eta) \widehat{v}(\gamma+i \eta)\|_{L^{2}\left(\mathbb{R}_{\eta}, U\right)}^{2} \leq C\|\widehat{v}(\lambda)\|_{L^{2}\left(\mathbb{R}_{\eta}, U\right)}^{2},
$$

for all compactly supported $v \in L^{2}(\mathbb{R}, U)$.

By density it follows that (3.11) holds for all $v \in L^{2}(\mathbb{R}, U)$. We have thus proved that (3.6) implies that $(\mathrm{H})$ holds true.

Proposition 3.3. Suppose that hypothesis (H) is satisfied. Then for $\left(x^{0}, x^{1}\right) \in V \times X$ we have that $B^{*} \phi($. $\in H^{1}(0, T ; U)$ and there exist $C, T>0$ such that the solution $\phi(t)$ of (2.3, 2.4) satisfies (3.3). In the other words assumption $(H)$ implies (3.3).

Proof. Suppose that hypothesis (H) is satisfied. Let $x(t) \in C(0, T ; V) \cap C^{1}(0, T ; X)$ be the unique solution of $(1.3,1.4)$. By Proposition 2.1 we know that $B^{*} x \in H^{1}(0, T ; U)$ and that $(2.1)$ holds true. Let $\phi$ be the solution of $(2.3,2.4)$. We clearly have $\psi=x-\phi \in C(0, T ; V) \cap C^{1}(0, T ; X)$ and $\psi$ satisfies

$$
\left\{\begin{array}{l}
\psi^{\prime \prime}(t)+A \psi(t)=B B^{*} x^{\prime}(t), \text { in } C\left(0, T ; V^{\prime}\right) \\
\psi(0)=\psi^{\prime}(0)=0
\end{array}\right.
$$

By applying now Proposition 3.2 with $v=B^{*} x^{\prime} \in L^{2}(0, T ; U)$ we obtain that

$$
\int_{0}^{T}\left\|\left(B^{*} \psi\right)^{\prime}(t)\right\|_{U}^{2} \mathrm{~d} t \leq C \int_{0}^{T}\left\|\left(B^{*} x\right)^{\prime}(t)\right\|_{U}^{2} \mathrm{~d} t
$$

Since $B^{*} \phi=B^{*} x-B^{*} \psi$ relations (2.1) and (3.12) imply the conclusion of the proposition.

Corollary 3.4. Suppose that assumption $(H)$ is satisfied. Then, for all $v \in L^{2}(0, T ; U)$, problem (3.1, 3.2) admits a unique solution y satisfying (3.5) and (3.6).

Proof. Suppose that assumption (H) is satisfied. Then Proposition 3.3 and Lemma 3.1 imply that problem (3.1, 3.2) admits a unique solution $y$ satisfying (3.5). Finally Proposition 3.2 implies that $y$ satisfies (3.6).

\section{Proof of the main Results}

Let $x(t) \in C(0, T ; V) \cap C^{1}(0, T ; X)$ be the solution of $(1.3,1.4)$. Then $x(t)$ can be written as

$$
x(t)=\phi(t)+\psi(t),
$$

where $\phi(t)$ satisfies $(2.3,2.4)$ and $\psi(t)$ satisfies

$$
\begin{gathered}
\psi^{\prime \prime}(t)+A \psi(t)=-B B^{*} x^{\prime}(t), \\
\psi(0)=\psi^{\prime}(0)=0 .
\end{gathered}
$$


The main ingredient of the proof of Theorem 2.2 and of the proof of Theorem 2.4 is the following result:

Lemma 4.1. Let $\left(x^{0}, x^{1}\right) \in V \times X$ and suppose that $(H)$ is verified. Then the solution $x(t)$ of $(1.3,1.4)$ and the solution $\phi(t)$ of (2.3, 2.4) satisfy

$$
C_{1} \int_{0}^{T}\left\|\left(B^{*} \phi\right)^{\prime}(t)\right\|_{U}^{2} \mathrm{~d} t \leq \int_{0}^{T}\left\|\left(B^{*} x\right)^{\prime}(t)\right\|_{U}^{2} \mathrm{~d} t \leq 4 \int_{0}^{T}\left\|\left(B^{*} \phi\right)^{\prime}(t)\right\|_{U}^{2} \mathrm{~d} t
$$

where $C_{1}>0$ is a constant independent of $\left(x^{0}, x^{1}\right)$.

Remark 4.2. By Proposition $2.1,\left(B^{*} x\right)^{\prime}(\cdot) \in L^{2}(0, T ; U)$. So, equation (4.2) makes sense. The result above shows that the $L^{2}$ norm of $\left\|\left(B^{*} x\right)^{\prime}(\cdot)\right\|_{U}$ is equivalent to the $L^{2}$ norm of $\left\|\left(B^{*} \phi\right)^{\prime}(\cdot)\right\|_{U}$ (notice that $\left\|\left(B^{*} \phi\right)^{\prime}(\cdot)\right\|_{U}$ $\in L^{2}(0, T)$ by Prop. 3.3).

Proof of Lemma 4.1. We prove (4.4) for $x(t)$ satisfying $(1.3,1.4)$ and $\phi(t)$ solution of $(2.3,2.4)$.

Relation (4.1) implies that

$$
\int_{0}^{T}\left\|\left(B^{*} \phi\right)^{\prime}(\cdot)\right\|_{U}^{2} \mathrm{~d} t \leq 2\left\{\int_{0}^{T}\left\|\left(B^{*} x\right)^{\prime}(\cdot)\right\|_{U}^{2} \mathrm{~d} t+\int_{0}^{T}\left\|\left(B^{*} \psi\right)^{\prime}(\cdot)\right\|_{U}^{2} \mathrm{~d} t\right\} .
$$

Estimate above combined with inequality (3.6) in Proposition 3.2 implies the existence of a constant $C_{1}>0$, independent of $\left(x^{0}, x^{1}\right)$, such that

$$
C_{1} \int_{0}^{T}\left\|\left(B^{*} \phi\right)^{\prime}(\cdot)\right\|_{U}^{2} \mathrm{~d} t \leq \int_{0}^{T}\left\|\left(B^{*} x\right)^{\prime}(\cdot)\right\|_{U}^{2} \mathrm{~d} t
$$

On the other hand, according to Remark 4.2 and to relation (4.1) we have that

$$
\left\|\left(B^{*} \phi\right)^{\prime}(\cdot)\right\|_{U} \in L^{2}(0, T) .
$$

This means that (4.2) can be rewritten as

$$
\psi^{\prime \prime}(t)+A \psi(t)+B\left(B^{*} \psi\right)^{\prime}(t)=-B\left(B^{*} \phi\right)^{\prime}(t) .
$$

We denote now by $w(t)$ the extension of $\left(B^{*} \phi\right)^{\prime}$ obtained by defining $w(t)=0, t \in \mathbb{R} \backslash[0, T]$. We still denote by $\psi(t)$ the solution of

$$
\left\{\begin{array}{l}
\psi^{\prime \prime}(t)+A \psi(t)+B\left(B^{*} \psi\right)^{\prime}=-B w(t), t \in \mathbb{R} \\
\psi(0)=\psi^{\prime}(0)=0
\end{array}\right.
$$

We clearly have $\psi(t)=0$ for $t \in \mathbb{R} \backslash[0, T]$.

Taking the Laplace transform we get

$$
\lambda^{2} \widehat{\psi}(\lambda)+A \widehat{\psi}(\lambda)+\lambda B B^{*} \widehat{\psi}(\lambda)=-B \widehat{w}(\lambda), \forall \lambda=\gamma+i \eta, \gamma>0 .
$$

The equality above holds in $\left(\mathcal{D}\left(A^{\frac{1}{2}}\right)\right)^{\prime}$.

By applying $\bar{\lambda} \overline{\hat{\psi}} \in \mathcal{D}\left(A^{\frac{1}{2}}\right)$ to the equality above, we get

$$
\lambda|\lambda|^{2}\|\widehat{\psi}(\lambda)\|_{X}^{2}+\bar{\lambda}\left\|A^{\frac{1}{2}} \widehat{\psi}(\lambda)\right\|_{X}^{2}+\left\|\lambda B^{*} \widehat{\psi}(\lambda)\right\|_{U}^{2}=-\left\langle w(\lambda), \bar{\lambda} B^{*} \overline{\hat{\psi}}(\lambda)\right\rangle_{U} .
$$

Taking the real part of each term, we get

$$
\int_{\mathbb{R}_{\eta}}\left\|\lambda B^{*} \widehat{\psi}(\lambda)\right\|_{U}^{2} \mathrm{~d} \eta \leq \frac{1}{2} \int_{\mathbb{R}_{\eta}}\|\widehat{w}(\lambda)\|_{U}^{2} \mathrm{~d} \eta+\frac{1}{2} \int_{\mathbb{R}_{\eta}}\left\|\lambda B^{*} \widehat{\psi}(\lambda)\right\|_{U}^{2} \mathrm{~d} \eta
$$


Parseval identity implies

$$
\left\|\left(B^{*} \psi\right)^{\prime}(t)\right\|_{L^{2}(0, T ; U)}^{2} \leq\left\|\left(B^{*} \phi\right)^{\prime}(t)\right\|_{L^{2}(0, T ; U)}^{2} .
$$

Relation (4.1) and inequality above imply that

$$
\left\|\left(B^{*} x\right)^{\prime}(t)\right\|_{L^{2}(0, T ; U)}^{2} \leq 4\left\|\left(B^{*} \phi\right)^{\prime}(t)\right\|_{L^{2}(0, T ; U)}^{2} .
$$

Inequalities (4.5) and (4.9) obviously yield the conclusion (4.4).

We can now prove the first main result.

Proof of Theorem 2.2. All finite energy solutions of $(1.3,1.4)$ satisfy the estimate

$$
E(x(t)) \leq M \mathrm{e}^{-\omega t} E(x(0)), \quad \forall t \geq 0,
$$

where $M, \omega>0$ are constants independent of $\left(x^{0}, x^{1}\right)$, if and only if there exist a time $T>0$ and a constant $C>0$ (depending on $T$ ) such that

$$
E(x(0))-E(x(T)) \geq C E(x(0)), \quad \forall\left(x^{0}, x^{1}\right) \in V \times X .
$$

By (2.1) relation above is equivalent to the inequality

$$
\int_{0}^{T}\left\|\left(B^{*} x\right)^{\prime}(s)\right\|_{U}^{2} \mathrm{~d} s \geq C E(x(0)), \quad \forall\left(x^{0}, x^{1}\right) \in V \times X .
$$

From Lemma 4.1 it follows that the system $(1.3,1.4)$ is exponentially stable if and only if

$$
\int_{0}^{T}\left\|\left(B^{*} \phi\right)^{\prime}(s)\right\|_{U}^{2} \mathrm{~d} s \geq C E(x(0)), \quad \forall\left(x^{0}, x^{1}\right) \in \mathcal{D}(A) \times V
$$

holds true. By density it follows that $(1.3,1.4)$ is exponentially stable if and only if $(2.5)$ holds true. This ends up the proof of Theorem 2.2.

Remark 4.3. By analyzing the proof above we notice that the proof of the inequality (4.9) does not require assumption (H). More precisely, the inequality (4.8) can be also obtained in the following direct manner:

Let $\left(x^{0}, x^{1}\right) \in V \times X$ such that $B^{*} \phi \in H^{1}(0, T ; U)$. Then, by formally multipying (4.7) by $\psi^{\prime}$, it follow that the function $\psi=x-\phi$ satisfies

$$
\|\left(\psi(t), \psi^{\prime}(t)\left\|_{V \times X}^{2}+\int_{0}^{t}\left(\left\|B^{*} \psi^{\prime}(s)\right\|_{U}^{2}+\left\|B^{*} x^{\prime}(s)\right\|_{U}^{2}\right) \mathrm{d} s=\int_{0}^{t}\right\| B^{*} \phi^{\prime}(s) \|_{U}^{2} \mathrm{~d} s .\right.
$$

This implies that (4.9) holds.

This means that the result mentioned in Remark 2.3 can be also established by a direct method.

Before giving the proof of Theorem 2.4, we need a technical lemma. This lemma extends a result in Jaffard et al. [11]. For a proof we refere to Ammari et al. [1].

Lemma 4.4. Let $\left(\mathcal{E}_{k}\right)$ be a sequence of positive real numbers satisfying

$$
\mathcal{E}_{k+1} \leq \mathcal{E}_{k}-C \mathcal{E}_{k+1}^{2+\alpha}, \forall k \geq 0
$$


where $C>0$ and $\alpha>-1$ are constants. Then there exists a positive constant $M$ such that

$$
\mathcal{E}_{k} \leq \frac{M}{(k+1)^{\frac{1}{1+\alpha}}}, \forall k \geq 0
$$

Proof of Theorem 2.4. By density (2.8) implies that for all $\left(x^{0}, x^{1}\right) \in V \times X$ we have

$$
\int_{0}^{T}\left\|\left(B^{*} \phi\right)^{\prime}(t)\right\|_{U}^{2} \mathrm{~d} t \geq C\left\|\left(x^{0}, x^{1}\right)\right\|_{V \times X}^{2} \mathcal{G}\left(\frac{\left\|\left(x^{0}, x^{1}\right)\right\|_{X_{1} \times X_{2}}^{2}}{\left\|\left(x^{0}, x^{1}\right)\right\|_{V \times X}^{2}}\right) .
$$

By applying Lemma 4.1 we obtain that the solution $x(t)$ of $(1.3,1.4)$ satisfies the following inequality

$$
\int_{0}^{T}\left\|\left(B^{*} x\right)^{\prime}(t)\right\|_{U}^{2} \mathrm{~d} t \geq C\left\|\left(x^{0}, x^{1}\right)\right\|_{V \times X}^{2} \mathcal{G}\left(\frac{\left\|\left(x^{0}, x^{1}\right)\right\|_{X_{1} \times X_{2}}^{2}}{\left\|\left(x^{0}, x^{1}\right)\right\|_{V \times X}^{2}}\right), \forall\left(x^{0}, x^{1}\right) \in V \times X .
$$

Relation above and (2.1) imply the existence of a constant $K>0$ such that

$$
\left\|\left(x(T), x^{\prime}(T)\right)\right\|_{V \times X}^{2} \leq\left\|\left(x^{0}, x^{1}\right)\right\|_{V \times X}^{2}-K\left\|\left(x^{0}, x^{1}\right)\right\|_{V \times X}^{2} \mathcal{G}\left(\frac{\left\|\left(x^{0}, x^{1}\right)\right\|_{X_{1} \times X_{2}}^{2}}{\left\|\left(x^{0}, x^{1}\right)\right\|_{V \times X}^{2}}\right), \quad \forall\left(x^{0}, x^{1}\right) \in \mathcal{D}\left(\mathcal{A}_{d}\right) .
$$

By using (2.7) (see again [24]), we obtain for fixed $\theta \in(0,1)$

$$
\frac{\left\|\left(x^{0}, x^{1}\right)\right\|_{X_{1} \times X_{2}}^{2}}{\left\|\left(x^{0}, x^{1}\right)\right\|_{V \times X}^{2}} \geq \frac{\left\|\left(x^{0}, x^{1}\right)\right\|_{X_{1} \times X_{2}}^{\frac{2-2 \theta}{\theta}}}{\left\|\left(x^{0}, x^{1}\right)\right\|_{V \times X}^{\frac{2-2 \theta}{\theta}}}, \forall\left(x^{0}, x^{1}\right) \in \mathcal{D}\left(\mathcal{A}_{d}\right)
$$

By using (4.14) combined with the fact that the function $t \rightarrow\left\|\left(x(t), x^{\prime}(t)\right)\right\|_{V \times X}^{2}$ is nonincreasing, the function $\mathcal{G}$ is increasing and relation (4.22) we obtain the existence of a constant $K_{1}>0$ such that

$$
\left\|\left(x(T), x^{\prime}(T)\right)\right\|_{V \times X}^{2} \leq\left\|\left(x^{0}, x^{1}\right)\right\|_{V \times X}^{2}-K_{1}\left\|\left(x^{0}, x^{1}\right)\right\|_{V \times X}^{2} \mathcal{G}\left(\frac{\left\|\left(x(T), x^{\prime}(T)\right)\right\|_{V \times X}^{\frac{2-2 \theta}{\theta}}}{\left\|\left(x^{0}, x^{1}\right)\right\|_{Y_{1} \times Y_{2}}^{\frac{2-2 \theta}{\theta}}}\right) .
$$

Estimate (4.15) remains valid in successive intervals $[k T,(k+1) T]$, so, we have

$$
\begin{gathered}
\left\|\left(x((k+1) T), x^{\prime}((k+1) T)\right)\right\|_{V \times X}^{2} \leq\left\|\left(x(k T), x^{\prime}(k T)\right)\right\|_{V \times X}^{2} \\
-K_{1}\left\|\left(x(k T), x^{\prime}(k T)\right)\right\|_{V \times X}^{2} \mathcal{G}\left(\frac{\left\|\left(x((k+1) T), x^{\prime}((k+1) T)\right)\right\|_{V \times X}^{\frac{2-2 \theta}{\theta}}}{\left\|\left(x(k T), x^{\prime}(k T)\right)\right\|_{Y_{1} \times Y_{2}}^{\frac{2-2 \theta}{\theta}}}\right) .
\end{gathered}
$$

Since $\mathcal{A}_{d}$ generates a semigroup of contractions in $\mathcal{D}\left(\mathcal{A}_{d}\right)$ and the graph norm on $\mathcal{D}\left(\mathcal{A}_{d}\right)$ is equivalent to $\|.\|_{Y_{1} \times Y_{2}}$, relations above imply the existence of a constant $K_{2}>0$ such that

$$
\left\|\left(x((k+1) T), x^{\prime}((k+1) T)\right)\right\|_{V \times X}^{2} \leq\left\|\left(x(k T), x^{\prime}(k T)\right)\right\|_{V \times X}^{2}
$$




$$
\begin{gathered}
-K_{2}\left\|\left(x(k T), x^{\prime}(k T)\right)\right\|_{V \times X}^{2} \mathcal{G}\left(\frac{\left\|\left(x((k+1) T), x^{\prime}((k+1) T)\right)\right\|_{V \times X}^{\frac{2-2 \theta}{\theta}}}{\left\|\left(x^{0}, x^{1}\right)\right\|_{\mathcal{D}\left(\mathcal{A}_{d}\right)}^{\frac{2-2 \theta}{\theta}}}\right), \\
\forall\left(x^{0}, x^{1}\right) \in \mathcal{D}\left(\mathcal{A}_{d}\right) .
\end{gathered}
$$

If we adopt now the notation

$$
\mathcal{E}_{k}=\mathcal{G}\left(\frac{\left\|\left(x(k T), x^{\prime}(k T)\right)\right\|_{V \times X}^{\frac{2-2 \theta}{\theta}}}{\left\|\left(x^{0}, x^{1}\right)\right\|_{\mathcal{D}\left(\mathcal{A}_{d}\right)}^{\frac{2-2 \theta}{\theta}}}\right)
$$

the inequalities (4.23) implies

$$
\frac{\left\|\left(x((k+1) T), x^{\prime}((k+1) T)\right)\right\|_{V \times X}^{2}}{\left\|\left(x(k T), x^{\prime}(k T)\right)\right\|_{V \times X}^{2}} \frac{\mathcal{E}_{k}}{\mathcal{E}_{k+1}} \mathcal{E}_{k+1} \leq \mathcal{E}_{k}-K_{2} \mathcal{E}_{k} \mathcal{E}_{k+1} .
$$

Since, the function $t \rightarrow\left\|\left(x(t), x^{\prime}(t)\right)\right\|_{V \times X}^{2}$ is nonincreasing and the function $\mathcal{G}$ is increasing, relation (4.18) implies

$$
\frac{\left\|\left(x((k+1) T), x^{\prime}((k+1) T)\right)\right\|_{V \times X}^{2}}{\left\|\left(x(k T), x^{\prime}(k T)\right)\right\|_{V \times X}^{2}} \frac{\mathcal{E}_{k}}{\mathcal{E}_{k+1}} \mathcal{E}_{k+1} \leq \mathcal{E}_{k}-K_{2} \mathcal{E}_{k+1}^{2} .
$$

According to (4.24), relation (4.19) gives,

$$
\begin{gathered}
\frac{\frac{1}{\left[\frac{\left\|\left(x(k T), x^{\prime}(k T)\right)\right\|_{V \times X}^{\frac{2-2 \theta}{\theta}}}{\left\|\left(x^{0}, x^{1}\right)\right\|_{\mathcal{D}\left(\mathcal{A}_{d}\right)}^{\frac{2-2 \theta}{\theta}}}\right]^{\frac{\theta}{1-\theta}}} \mathcal{G}\left(\frac{\left\|\left(x(k T), x^{\prime}(k T)\right)\right\|_{V \times X}^{\frac{2-2 \theta}{\theta}}}{\left\|\left(x^{0}, x^{1}\right)\right\|_{\mathcal{D}\left(\mathcal{A}_{d}\right)}^{\frac{2-2 \theta}{\theta}}}\right)}{\frac{1}{\left[\frac{\left\|\left(x((k+1) T), x^{\prime}((k+1) T)\right)\right\|_{V \times X}}{\frac{2-2 \theta}{\theta}}\right]^{\frac{\theta}{1-\theta}}} \mathcal{G}\left(\frac{\left\|\left(x((k+1) T), x^{\prime}((k+1) T)\right)\right\|_{V \times X}^{\frac{2-2 \theta}{\theta}}}{\left\|\left(x^{0}, x^{1}\right)\right\|_{\mathcal{D}\left(\mathcal{A}_{d}\right)}^{\frac{2-2 \theta}{\theta}}}\right)} \mathcal{E}_{k+1} \\
\leq \mathcal{E}_{k}-K_{2} \mathcal{E}_{k+1}^{2} .
\end{gathered}
$$

Relation (4.20) combined with that the function $x \rightarrow \frac{1}{x^{\frac{\theta}{1-\theta}}} \mathcal{G}(x)$ is increasing in $(0,1)$, gives

$$
\mathcal{E}_{k+1} \leq \mathcal{E}_{k}-K_{2} \mathcal{E}_{k+1}^{2}, \forall k \geq 0
$$

By applying Lemma 4.4 and using relation (4.24) we obtain the existence of a constant $M>0$ such that

$$
\left\|\left(x(k T), x^{\prime}(k T)\right)\right\|_{V \times X}^{2} \leq\left[\mathcal{G}^{-1}\left(\frac{M}{k+1}\right)\right]^{\frac{\theta}{1-\theta}}\left\|\left(x^{0}, x^{1}\right)\right\|_{\mathcal{D}\left(\mathcal{A}_{d}\right)}^{2}, \quad \forall k \geq 0
$$

which obviously implies (2.9). 
Proof of second assertion of Theorem 2.4. By density (2.10) implies that for all $\left(x^{0}, x^{1}\right) \in V \times X$ we have

$$
\int_{0}^{T}\left\|\left(B^{*} \phi\right)^{\prime}(t)\right\|_{U}^{2} \mathrm{~d} t \geq C\left\|\left(x^{0}, x^{1}\right)\right\|_{X_{1} \times X_{2}}^{2} .
$$

Then, Lemma 4.1 combined with (2.7) and (2.1) imply the existence of a constant $K>0$ such that

$$
\begin{gathered}
\left\|\left(x(T), x^{\prime}(T)\right)\right\|_{V \times X}^{2} \leq \\
\forall\left(x^{0}, x^{1}\right) \|_{V \times X}^{2}-K \frac{\left\|\left(x^{0}, x^{1}\right)\right\|_{X_{1} \times X_{2}}^{\frac{2}{\theta}}}{\left\|\left(x^{0}, x^{1}\right)\right\|_{V \times X}^{\frac{2-2 \theta}{\theta}}}, \\
\forall\left(x^{0}, x^{1}\right) \in \mathcal{D}\left(\mathcal{A}_{d}\right) .
\end{gathered}
$$

Following the same steps as in the proof of the first assertion of Theorem 2.4 we obtain the existence of a constant $C>0$ such that for all $k \geq 0$ we have

$$
\begin{gathered}
\left\|\left(x((k+1) T), x^{\prime}((k+1) T)\right)\right\|_{V \times X}^{2} \leq\left\|\left(x(k T), x^{\prime}(k T)\right)\right\|_{V \times X}^{2} \\
-C \frac{\left\|\left(x((k+1) T), x^{\prime}((k+1) T)\right)\right\|_{V \times X}^{\frac{2}{\theta}}}{\left\|\left(x^{0}, x^{1}\right)\right\|_{\mathcal{D}\left(\mathcal{A}_{d}\right)}^{\frac{2-2 \theta}{\theta}}}, \quad \forall\left(x^{0}, x^{1}\right) \in \mathcal{D}\left(\mathcal{A}_{d}\right) .
\end{gathered}
$$

If we adopt the notation

$$
\mathcal{H}_{k}=\frac{\left\|\left(x(k T), x^{\prime}(k T)\right)\right\|_{V \times X}^{2}}{\left\|\left(x^{0}, x^{1}\right)\right\|_{\mathcal{D}\left(\mathcal{A}_{d}\right)}^{2}}
$$

relation (4.23) gives

$$
\mathcal{H}_{k+1} \leq \mathcal{H}_{k}-C \mathcal{H}_{k+1}^{\frac{1}{\theta}}, \forall k \geq 0
$$

By applying Lemma 4.4 and using relation (4.25) we obtain the existence of a constant $M>0$ such that

$$
\left\|\left(x(k T), x^{\prime}(k T)\right)\right\|_{V \times X}^{2} \leq \frac{M\left\|\left(x^{0}, x^{1}\right)\right\|_{\mathcal{D}\left(\mathcal{A}_{d}\right)}^{2}}{(k+1)^{\frac{\theta}{1-\theta}}}, \forall k \geq 0,
$$

which obviously implies (2.10).

\section{SOME APPLiCATiOnS}

Now, we give some applications of Theorem 2.2 and Theorem 2.4. Some of them are new and some were obtained by different methods in previous literature.

\subsection{First example: Stabilization of the string}

We consider the following initial and boundary problem:

$$
(I)\left\{\begin{array}{l}
\frac{\partial^{2} u}{\partial t^{2}}-\frac{\partial^{2} u}{\partial x^{2}}+\frac{\partial u}{\partial t}(\xi, t) \delta_{\xi}=0,(x, t) \in(0,1) \times(0,+\infty), \\
u(0, t)=u(1, t)=0, t \in(0,+\infty) \\
u(x, 0)=u^{0}(x), \frac{\partial u}{\partial t}(x, 0)=u^{1}(x), x \in(0,1),
\end{array}\right.
$$


where $\xi \in(0,1)$ and $\delta_{\xi}$ is the Dirac mass concentrated in the point $\xi \in(0,1)$.

In this case, we have:

$$
X=L^{2}(0,1), U=\mathbb{R}, V=H_{0}^{1}(0,1)
$$

and

$$
A=-\frac{\mathrm{d}^{2}}{\mathrm{~d} x^{2}}, \mathcal{D}(A)=H^{2}(0,1) \cap H_{0}^{1}(0,1), B k=k \delta_{\xi}, \forall k \in \mathbb{R}
$$

Then, $\mathcal{A}_{d}$ is given by

$$
\begin{gathered}
\mathcal{A}_{d}\left(\begin{array}{l}
u \\
v
\end{array}\right)=\left(\begin{array}{c}
v \\
\frac{\mathrm{d}^{2} u}{\mathrm{~d} x^{2}}-v(\xi) \delta_{\xi}
\end{array}\right), \\
\forall(u, v) \in \mathcal{D}\left(\mathcal{A}_{d}\right)=\left\{(u, v) \in\left[H_{0}^{1}(0,1) \cap H^{2}(0, \xi) \cap H^{2}(\xi, 1)\right] \times H_{0}^{1}(0,1), \quad \frac{\mathrm{d} u}{\mathrm{~d} x}\left(\xi^{+}\right)-\frac{\mathrm{d} u}{\mathrm{~d} x}\left(\xi^{-}\right)=v(\xi)\right\} .
\end{gathered}
$$

Denote by $Q$ the set of all rational numbers. Let us also denote by $\mathcal{S}$ the set of all numbers $\rho \in(0,1)$ such that $\rho \notin Q$ and if $\left[0, a_{1}, \ldots, a_{n}, \ldots\right]$ is the expansion of $\rho$ as a continued fraction, then $\left(a_{n}\right)$ is bounded. Let us notice that $\mathcal{S}$ is is obviously uncountable and, by classical results on diophantine approximation ( $c f$. [7], p. 120), its Lebesgue measure is equal to zero. In particular, by Euler-Lagrange theorem (see Lang [15], p. 57) $\mathcal{S}$ contains all $\xi \in(0,1)$ such that $\xi$ is an irrational quadratic number (i.e. satisfying a second degree equation with rational coefficients). According to a classical result (see for instance Tucsnak [25] and the references therein) if $\xi \in \mathcal{S}$ then there exists a constant $C_{\xi}>0$ such that

$$
|\sin (n \pi \xi)| \geq \frac{C_{\xi}}{n}, \quad \forall n \geq 1 .
$$

Stability results for (I) are then an immediate consequence of Theorem 2.2 and Theorem 2.4. We have the following result:

Theorem 5.1. 1. For any $\xi \in(0,1)$ the system described by $(I)$ is not exponentially stable in $V \times L^{2}(0,1)$.

2. For all $\xi \in \mathcal{S}$ and for all $t \geq 0$ we have

$$
\begin{gathered}
\left\|\left(u(t), \frac{\partial u}{\partial t}(t)\right)\right\|_{V \times L^{2}(0,1)}^{2} \leq \frac{C_{\xi}}{t+1}\left\|\left(u^{0}, u^{1}\right)\right\|_{\mathcal{D}\left(\mathcal{A}_{d}\right)}^{2}, \\
\forall\left(u^{0}, u^{1}\right) \in \mathcal{D}\left(\mathcal{A}_{d}\right),
\end{gathered}
$$

where $C_{\xi}>0$ is a constant depending only on $\xi$.

3. If $\epsilon>0$ then, for almost all $\xi \in(0,1)$ and for all $t \geq 0$ we have

$$
\left\|\left(u(t), \frac{\partial u}{\partial t}(t)\right)\right\|_{V \times L^{2}(0,1)}^{2} \leq \frac{C_{\xi, \epsilon}}{(t+1)^{\frac{1}{1+\epsilon}}}\left\|\left(u^{0}, u^{1}\right)\right\|_{\mathcal{D}\left(\mathcal{A}_{d}\right)}^{2},
$$




$$
\forall\left(u^{0}, u^{1}\right) \in \mathcal{D}\left(\mathcal{A}_{d}\right),
$$

where $C_{\xi, \epsilon}>0$ is a constant depending only on $\xi$ and $\epsilon$.

Remark 5.2. The first assertion of the theorem above was proved by a different method in Bamberger et al. [3]. In this case the problem $(2.3,2.4)$ becomes

$$
\begin{gathered}
\frac{\partial^{2} \phi}{\partial t^{2}}-\frac{\partial^{2} \phi}{\partial x^{2}}=0,(0,1) \times(0,+\infty), \\
\phi(0, t)=\phi(1, t)=0,(0,+\infty), \\
\phi(x, 0)=u^{0}(x), \frac{\partial \phi}{\partial t}(x, 0)=u^{1}(x),(0,1) .
\end{gathered}
$$

Lemma 5.3. The operators $A$ and $B$ defined by (5.1) satisfy assumption (H).

Proof. Let $k \in \mathbb{R}$. It can be easily cheked that $v=\left(\lambda^{2}+A\right)^{-1} B k$ satisfies:

$$
\begin{gathered}
\lambda^{2} v(x)-\frac{\mathrm{d}^{2} v}{\mathrm{~d} x^{2}}(x)=0, x \in(0, \xi) \cup(\xi, 1), \operatorname{Re} \lambda>0, \\
v(0)=v(1)=0, \\
{[v]_{\xi}=0,\left[\frac{\mathrm{d} v}{\mathrm{~d} x}\right]_{\xi}=k,}
\end{gathered}
$$

where we denote by $[g]$ the jump of the function $g$ at the point $\xi$.

The solutions of $(5.8,5.9)$ have the form

$$
v(x)=\left\{\begin{array}{l}
A \operatorname{sh}(\lambda x), x \in(0, \xi) \\
B \operatorname{sh}[\lambda(x-1)], x \in(\xi, 1)
\end{array}\right.
$$

where $A, B$ are constants.

Consequently, the solutions of (5.8-5.10) have the following form

$$
v(x)=\left\{\begin{array}{l}
\frac{1}{\lambda} \frac{\operatorname{sh}[\lambda(\xi-1)] \operatorname{sh}(\lambda x)}{\operatorname{sh}(\lambda)} k, x \in(0, \xi), \\
\frac{1}{\lambda} \frac{\operatorname{sh}(\lambda \xi) \operatorname{sh}[\lambda(x-1)]}{\operatorname{sh}(\lambda)} k, x \in(\xi, 1) .
\end{array}\right.
$$

Then, the function $H(\lambda)=\lambda B^{*}\left(\lambda^{2}+A\right)^{-1} B$ associated to problem (I) is given by the following expression

$$
H(\lambda)=\frac{\operatorname{sh}(\lambda \xi) \operatorname{sh}[\lambda(\xi-1)]}{\operatorname{sh}(\lambda)}, \operatorname{Re} \lambda>0 .
$$


We easily check that

$$
\sup _{\lambda \in C_{\beta}}|H(\lambda)| \leq \frac{\operatorname{ch}(\beta \xi) \operatorname{ch}[\beta(\xi-1)]}{\operatorname{sh}(\beta)}
$$

Thus $(\mathrm{H})$ is satisfied.

The observability inequality concerning the trace at the point $x=\xi$ of the solutions of $(5.5-5.7)$ is given in the proposition below.

Proposition 5.4. Let $T>0$ be fixed. Then the following assertions hold true.

1. For all $\xi \in \mathcal{S}$ the solution $\phi$ of (5.5-5.7) satisfies

$$
\begin{gathered}
\int_{0}^{T}\left[\frac{\partial \phi}{\partial t}(\xi, t)\right]^{2} \mathrm{~d} t \geq C_{\xi}\left(\left\|u^{0}\right\|_{L^{2}(0,1)}^{2}+\left\|u^{1}\right\|_{H^{-1}(0,1)}^{2}\right), \\
\forall\left(u^{0}, u^{1}\right) \in V \times L^{2}(0,1),
\end{gathered}
$$

where $C_{\xi}>0$ is a constant depending only on $\xi$.

2. For all $\epsilon>0$ and for almost all $\xi \in(0,1)$ the solution $\phi$ of (5.5-5.7) satisfies

$$
\begin{gathered}
\int_{0}^{T}\left[\frac{\partial \phi}{\partial t}(\xi, t)\right]^{2} \mathrm{~d} t \geq C_{\xi, \epsilon}\left(\left\|u^{0}\right\|_{H^{-\epsilon}(0,1)}^{2}+\left\|u^{1}\right\|_{H^{-1-\epsilon}(0,1)}^{2}\right), \\
\forall\left(u^{0}, u^{1}\right) \in V \times L^{2}(0,1)
\end{gathered}
$$

where $C_{\xi, \epsilon}>0$ is a constant depending only on $\xi$ and $\epsilon$.

3. The result in assertion 1 is sharp in the sense that, for all $\xi \in(0,1)$, there exists a sequence $\left(u_{m}^{0}, u_{m}^{1}\right)$ $\subset V \times L^{2}(0,1)$ such that the corresponding sequence of solutions $\left(\phi_{m}\right)$ of (5.5-5.7) with initial data $\left(u_{m}^{0}, u_{m}^{1}\right)$ satisfies

$$
\lim _{m \rightarrow \infty} \frac{\int_{0}^{T}\left[\frac{\partial \phi}{\partial t}(\xi, t)\right]^{2} \mathrm{~d} t}{\left\|u^{0}\right\|_{H^{\epsilon}(0,1)}^{2}+\left\|u^{1}\right\|_{H^{-1+\epsilon}(0,1)}^{2}}=0
$$

Proof. If we put

$$
u^{0}(x)=\sum_{n=1}^{\infty} a_{n} \sin (n \pi x), u^{1}(x)=\sum_{n=1}^{\infty} n b_{n} \sin (n \pi x)
$$

with

then we clearly have

$$
\left(n a_{n}\right),\left(n b_{n}\right) \subset l^{2}(\mathbb{R}),
$$

$$
\frac{\partial \phi}{\partial t}(\xi, t)=\sum_{n \geq 1}\left(-n \pi a_{n} \sin (n \pi t) \sin (n \pi \xi)+n \pi b_{n} \cos (n \pi t) \sin (n \pi \xi)\right) .
$$


From Ingham's inequality (see Ingham [10]) we obtain, for all $T>2$, the existence of a constant $C_{T}>0$ such that the solution $\phi$ of $(5.5-5.7)$ satisfies

$$
\int_{0}^{T}\left[\frac{\partial \phi}{\partial t}(\xi, t)\right]^{2} \mathrm{~d} t \geq C_{T} \sum_{n \geq 1} n^{2}\left(a_{n}^{2}+b_{n}^{2}\right)|\sin (n \pi \xi)|^{2} .
$$

Relations (5.16) and (5.2) imply the existence of a constant $K_{T, \xi}>0$ such that

$$
\int_{0}^{T}\left[\frac{\partial \phi}{\partial t}(\xi, t)\right]^{2} \mathrm{~d} t \geq K_{T, \xi} \sum_{n=0}^{\infty}\left[a_{n}^{2}+b_{n}^{2}\right], \quad \forall \xi \in \mathcal{S},
$$

which is exactly (5.11).

In order to prove (5.12) we use a well-known result asserting that, for all $\epsilon>0$ there exists a set $B_{\epsilon} \subset(0,1)$ having the Lebesgue measure equal to 1 and a constant $C>0$, such that for any, $\rho \in B_{\epsilon}$,

$$
|\sin (n \pi \rho)| \geq \frac{C}{n^{1+\epsilon}}, \quad \forall n \geq 1 .
$$

Let us notice that by Roth's theorem $B_{\epsilon}$ contains all numbers in $(0,1)$ having the property that $\xi$ is an algebraic irrational (see Cassals [7] for details). Inequalities (5.16) and (5.17) obviously imply (5.12).

We still have to show the existence of a sequence satisfying (5.13). By using continous fractions (see again [25] and references therein for details) we construct a sequence $\left(q_{m}\right) \subset \mathbb{N}$ such that $q_{m} \rightarrow \infty$ and

$$
\left|\sin \left(q_{m} \pi \xi\right)\right| \leq \frac{\pi}{q_{m}}, \forall m \geq 1
$$

Using (5.15) and (5.47) a simple calculation shows that the sequence $\left(\phi_{m}^{0}, \phi_{m}^{1}\right)=\left(\sin \left(q_{m} \pi x\right), 0\right)$ satisfies $(5.13)$.

Proof of Theorem 5.1. According to Theorem 2.2, the solutions of (I) satisfy the estimate

$$
\left\|\left(u(t), \frac{\partial u}{\partial t}(t)\right)\right\|_{V \times L^{2}(0,1)}^{2} \leq M \mathrm{e}^{-\omega t}\left\|\left(u^{0}, u^{1}\right)\right\|_{V \times L^{2}(0,1)}^{2}, \quad \forall t \geq 0,
$$

where $M, \omega>0$ are constants depending only on $\xi$, if and only if the solution $\phi$ of $(5.5-5.7)$ satisfies

$$
\int_{0}^{T}\left|\frac{\partial \phi}{\partial t}(\xi, s)\right|^{2} \mathrm{~d} s \geq C\left\|\left(u^{0}, u^{1}\right)\right\|_{V \times L^{2}(0,1)}^{2}, \quad \forall\left(u^{0}, u^{1}\right) \in V \times L^{2}(0,1) .
$$

The inequality above clearly contradicts assertion 3 in Proposition 5.4. So assumption (5.38) is false. We end up in this way the proof of the first assertion of theorem.

We pass now to the proof of the second assertion of this theorem. Let $\xi \in \mathcal{S}$. By Proposition 5.4, the solution $\phi$ of (5.5-5.7) satisfies the inequality

$$
\int_{0}^{T}\left|\frac{\partial \phi}{\partial t}(\xi, t)\right|^{2} \mathrm{~d} t \geq K_{1}\left(\left\|u^{0}\right\|_{L^{2}(0,1)}^{2}+\left\|u^{1}\right\|_{H^{-1}(0,1)}^{2}\right), \forall\left(u^{0}, u^{1}\right) \in V \times L^{2}(0,1)
$$

where $K_{1}>0$ is a constant. The conclusion (5.3) follows now by simply using the Theorem 2.4 (with $X_{1}$ $\left.=L^{2}(0,1), X_{2}=H^{-1}(0,1), Y_{1} \times Y_{2}=\left[H_{0}^{1}(0,1) \cup H^{2}(0, \xi) \cup H^{2}(\xi, 1)\right] \times H_{0}^{1}(0,1), \mathcal{G}(x)=x, \forall x \in \mathbb{R}^{+}, \theta=\frac{1}{2}\right)$. 
Let us now suppose that $\epsilon>0$ and that $\xi$ belongs to the set $B_{\epsilon}$. From (5.12), it follows (5.4) by Theorem 2.4 (with $X_{1}=H^{-\epsilon}(0,1), X_{2}=H^{-1-\epsilon}(0,1), Y_{1} \times Y_{2}=\left[H_{0}^{1}(0,1) \cup H^{2}(0, \xi) \cup H^{2}(\xi, 1)\right] \times H_{0}^{1}(0,1), \mathcal{G}(x)=x, \forall x$ $\left.\in \mathbb{R}^{+}, \theta=\frac{1}{2+\epsilon}\right)$.

Remark 5.5. The same method can be applied for a string with different boundary conditions (see Ammari et al. [2]).

\subsection{Second example: Stabilization of the Bernoulli-Euler beam}

We consider the following initial and boundary value problem:

$$
\begin{gathered}
\frac{\partial^{2} u}{\partial t^{2}}(x, t)+\frac{\partial^{4} u}{\partial x^{4}}(x, t)+\frac{\partial u}{\partial t}(\xi, t) \delta_{\xi}=0, \quad 0<x<1, t>0 \\
u(0, t)=u(1, t)=\frac{\partial^{2} u}{\partial x^{2}}(0, t)=\frac{\partial^{2} u}{\partial x^{2}}(1, t)=0, \quad t>0 \\
u(x, 0)=u^{0}(x), \frac{\partial u}{\partial t}(x, 0)=u^{1}(x), \quad 0<x<1 .
\end{gathered}
$$

Here $u$ denotes the transverse displacement of the beam, $\delta_{\xi}$ is the Dirac mass concentrated at the point $\xi \in(0,1)$ and we suppose that the length of the beam is equal to 1 .

In this case,

$$
X=L^{2}(0,1), U=\mathbb{R}, V=H^{2}(0,1) \cap H_{0}^{1}(0,1)
$$

and

$$
\begin{aligned}
& A=\frac{\mathrm{d}^{4}}{\mathrm{~d} x^{4}}, \mathcal{D}(A)=\left\{u \in H^{4}(0,1) \cap H_{0}^{1}(0,1), \frac{\mathrm{d}^{2} u}{\mathrm{~d} x^{2}}(0)=\frac{\mathrm{d}^{2} u}{\mathrm{~d} x^{2}}(1)=0\right\} \\
& B k=k \delta_{\xi}, \forall k \in \mathbb{R}
\end{aligned}
$$

$\mathcal{A}_{d}$ is given by

$$
\begin{gathered}
\mathcal{A}_{d}\left(\begin{array}{l}
u \\
v
\end{array}\right)=\left(\begin{array}{c}
v \\
-\frac{\mathrm{d}^{4} u}{\mathrm{~d} x^{4}}-v(\xi) \delta_{\xi}
\end{array}\right), \\
\forall(u, v) \in \mathcal{D}\left(\mathcal{A}_{d}\right)=\left\{(u, v) \in\left[H^{2}(0,1) \cap H^{4}(0, \xi) \cap H^{4}(\xi, 1)\right] \times H^{2}(0,1), \quad u(0)=v(0)\right. \\
\left.=u(1)=v(1)=\frac{\mathrm{d}^{2} u}{\mathrm{~d} x^{2}}(0)=\frac{\mathrm{d}^{2} u}{\mathrm{~d} x^{2}}(1)=0, \frac{\mathrm{d}^{2} u}{\mathrm{~d} x^{2}}(\xi+)=\frac{\mathrm{d}^{2} u}{\mathrm{~d} x^{2}}(\xi-), \frac{\mathrm{d}^{3} u}{\mathrm{~d} x^{3}}(\xi+)-\frac{\mathrm{d}^{3} u}{\mathrm{~d} x^{3}}(\xi-)=-v(\xi)\right\} .
\end{gathered}
$$

The stability results can now be stated as follows.

Theorem 5.6. 1. For any $\xi \in(0,1)$ the system described by (5.20-5.22) is not exponentially stable in $V \times L^{2}(0,1)$. 
2. For all $\xi$ in the set $\mathcal{S}$ defined in Section 5.1 and for all $t \geq 0$ we have

$$
\begin{gathered}
\left\|\left(u(t), \frac{\partial u}{\partial t}(t)\right)\right\|_{V \times L^{2}(0,1)}^{2} \leq \frac{C_{\xi}}{(t+1)^{2}}\left\|\left(u^{0}, u^{1}\right)\right\|_{\mathcal{D}\left(\mathcal{A}_{d}\right)}^{2}, \\
\forall\left(u^{0}, u^{1}\right) \in \mathcal{D}\left(\mathcal{A}_{d}\right),
\end{gathered}
$$

where $C_{\xi}>0$ is a constant depending only on $\xi$.

3. If $\epsilon>0$ then, for almost all $\xi \in(0,1)$ and for all $t \geq 0$ we have

$$
\begin{gathered}
\left\|\left(u(t), \frac{\partial u}{\partial t}(t)\right)\right\|_{V \times L^{2}(0,1)}^{2} \leq \frac{C_{\xi, \epsilon}}{(t+1)^{\frac{2}{1+\epsilon}}}\left\|\left(u^{0}, u^{1}\right)\right\|_{\mathcal{D}\left(\mathcal{A}_{d}\right)}^{2}, \\
\forall\left(u^{0}, u^{1}\right) \in \mathcal{D}\left(\mathcal{A}_{d}\right),
\end{gathered}
$$

where $C_{\xi, \epsilon}>0$ is a constant depending only on $\xi$ and $\epsilon$.

In this case the problem $(2.3,2.4)$ becomes

$$
\begin{gathered}
\frac{\partial^{2} \phi}{\partial t^{2}}(x, t)+\frac{\partial^{4} \phi}{\partial x^{4}}(x, t)=0, \quad 0<x<1, t>0, \\
\phi(0, t)=\phi(1, t)=\frac{\partial^{2} \phi}{\partial x^{2}}(0, t)=\frac{\partial^{2} \phi}{\partial x^{2}}(1, t)=0, \quad t>0, \\
\phi(x, 0)=u^{0}(x), \frac{\partial \phi}{\partial t}(x, 0)=u^{1}(x), \quad 0<x<1 .
\end{gathered}
$$

Lemma 5.7. The operators $A$ and $B$ defined in (5.23) satisfy assumption (H).

Proof. Let $k \in \mathbb{R}$. It can be easily checked that $v=\left(\lambda^{2}-A\right)^{-1} B k$ satisfies:

$$
\begin{gathered}
\lambda^{2} v(x)+\frac{\mathrm{d}^{4} v}{\mathrm{~d} x^{4}}(x)=0, x \in(0, \xi) \cup(\xi, 1), \operatorname{Re} \lambda>0 \\
v(0)=v(1)=\frac{\mathrm{d}^{2} v}{\mathrm{~d} x^{2}}(0)=\frac{\mathrm{d}^{2} v}{\mathrm{~d} x^{2}}(1)=0, \operatorname{Re} \lambda>0, \\
{[v]_{\xi}=\left[\frac{\mathrm{d} v}{\mathrm{~d} x}\right]_{\xi}=\left[\frac{\mathrm{d}^{2} v}{\mathrm{~d} x^{2}}\right]_{\xi}=0,} \\
{\left[\frac{\mathrm{d}^{3} v}{\mathrm{~d} x^{3}}\right]_{\xi}=k, \operatorname{Re} \lambda>0}
\end{gathered}
$$

where we denote by $[f]_{\xi}$ the jump of the function $f$ at the point $\xi$. 
Recall that $\lambda=i w^{2}$ where $w \in\left\{r \mathrm{e}^{i \gamma} ; r>0, \gamma \in\right]-\frac{\pi}{2}, 0[\}$. Then, the solutions of (5.29-5.31) have the following form

$$
v(x)=\left\{\begin{array}{l}
-\frac{1}{2 w^{3}}\left\{\frac{\sin [w(\xi-1)] \sin (w x)}{\sin (w)}-\frac{\operatorname{sh}(w x) \operatorname{sh}[w(\xi-1)]}{\operatorname{sh}(w)}\right\} k, x \in(0, \xi), \\
-\frac{1}{2 w^{3}}\left\{\frac{\sin (w \xi) \sin [(w(x-1)])}{\sin (w)}-\frac{\operatorname{sh}(w \xi) \operatorname{sh}[w(x-1)]}{\operatorname{sh}(w)}\right\} k, x \in(\xi, 1) .
\end{array}\right.
$$

Then the function $H(\lambda)=\lambda B^{*}\left(\lambda^{2}-A\right)^{-1} B$ is given by

$$
H\left(\lambda=i w^{2}\right)=\frac{i}{2 w}\left\{-\frac{\sin (w \xi) \sin [w(\xi-1)]}{\sin (w)}+\frac{\operatorname{sh}(w \xi) \operatorname{sh}[w(\xi-1)]}{\operatorname{sh}(w)}\right\}, \forall \operatorname{Re} \lambda>0
$$

By Lemma 3.4 in [1] we conclude that $H(\lambda)$ is bounded on $C_{\beta}$.

The observability inequalities are given in the proposition below.

Proposition 5.8. Let $T>0$ be fixed and $\mathcal{S} \subset[0,1]$ be the set introduced in Section 5.1. Then

1. For all $\xi \in \mathcal{S}$ the solution $\phi$ of (5.26-5.28) satisfies

$$
\begin{gathered}
\int_{0}^{T}\left|\frac{\partial \phi}{\partial t}(\xi, t)\right|^{2} \mathrm{~d} t \geq C_{\xi}\left(\left\|u^{0}\right\|_{H^{1}(0,1)}^{2}+\left\|u^{1}\right\|_{H^{-1}(0,1)}^{2}\right), \\
\forall\left(u^{0}, u^{1}\right) \in V \times L^{2}(0,1)
\end{gathered}
$$

where $C_{\xi}>0$ is a constant depending only on $\xi$.

2. For all $\epsilon>0$ and for almost all $\xi \in(0,1)$ the solution $\phi$ of (5.26-5.28) satisfies

$$
\begin{gathered}
\int_{0}^{T}\left|\frac{\partial \phi}{\partial t}(\xi, t)\right|^{2} \mathrm{~d} t \geq C_{\xi, \epsilon}\left(\left\|u^{0}\right\|_{H^{1-\epsilon}(0,1)}^{2}+\left\|u^{1}\right\|_{H^{-1-\epsilon}(0,1)}^{2}\right), \\
\forall\left(u^{0}, u^{1}\right) \in V \times L^{2}(0,1)
\end{gathered}
$$

where $C_{\xi, \epsilon}>0$ is a constant depending only on $\xi$ and $\epsilon$.

3. The result in assertion 1 is sharp in the sense that, for all $\xi \in(0,1)$, there exists a sequence $\left(u_{m}^{0}, u_{m}^{1}\right)$ $\subset V \times L^{2}(0,1)$ such that the corresponding sequence of solutions $\left(\phi_{m}\right)$ of $(5.26,5.27)$ with initial data $\left(u_{m}^{0}, u_{m}^{1}\right)$ satisfies

$$
\lim _{m \rightarrow \infty} \frac{\int_{0}^{T}\left|\frac{\partial \phi_{m}}{\partial t}(\xi, t)\right|^{2} \mathrm{~d} t}{\left\|u_{m}^{0}\right\|_{H^{1+\epsilon}(0,1)}^{2}+\left\|u_{m}^{1}\right\|_{H^{-1+\epsilon}(0,1)}^{2}}=0 .
$$

Proof. Let

$$
u^{0}(x)=\sum_{n=1}^{\infty} a_{n} \sin (n \pi x), u^{1}(x)=\sum_{n=1}^{\infty} n b_{n} \sin (n \pi x)
$$


with

Which implies that

$$
\left(n^{2} a_{n}\right),\left(n b_{n}\right) \subset l^{2}(\mathbb{R})
$$

$$
\frac{\partial \phi}{\partial t}(\xi, t)=\sum_{n=1}^{\infty}\left[-n^{2} \pi^{2} a_{n} \sin \left(n^{2} \pi^{2} t\right) \sin (n \pi \xi)+n^{2} \pi^{2} b_{n} \cos \left(n^{2} \pi^{2} t\right) \sin (n \pi \xi)\right] .
$$

From (5.36) and Ingham's inequality (see [10]), we obtain that, for all $T>2$, there exists a constant $C_{T}>0$ such that

$$
\int_{0}^{T}\left|\frac{\partial \phi}{\partial t}(\xi, t)\right|^{2} \mathrm{~d} t \geq C_{T} \sum_{n=1}^{\infty}\left[n^{4} a_{n}^{2} \sin ^{2}(n \pi \xi)+n^{4} b_{n}^{2} \sin ^{2}(n \pi \xi)\right] .
$$

Suppose now that $\xi$ belongs to the set $\mathcal{S}$ defined in Section 5.1. Then relations (5.37) and (5.2) imply the existence of a constant $K_{T, \xi}>0$ such that

$$
\int_{0}^{T}\left|\frac{\partial \phi}{\partial t}(\xi, t)\right|^{2} \mathrm{~d} t \geq K_{T, \xi} \sum_{n=1}^{\infty}\left[n^{2} a_{n}^{2}+n^{2} b_{n}^{2}\right], \quad \forall \xi \in \mathcal{S}
$$

which is exactly (5.33).

Assertions 2 and 3 of the proposition can be proved by simply adapting the proof of Proposition 5.4. So we skip the details.

Proof of Theorem 5.6. 1. According to Theorem 2.2, all finite energy solutions of (5.20-5.22) satisfy the estimate

$$
\left\|\left(u(t), \frac{\partial u}{\partial t}(t)\right)\right\|_{V \times L^{2}(0,1)}^{2} \leq M \mathrm{e}^{-\omega t}\left\|\left(u^{0}, u^{1}\right)\right\|_{V \times L^{2}(0,1)}^{2}, \quad \forall t \geq 0,
$$

where $M, \omega>0$ are constants depending only on $\xi$, if and only if the solution $\phi$ of (5.26-5.28) satisfies

$$
\int_{0}^{T}\left|\frac{\partial \phi}{\partial t}(\xi, s)\right|^{2} \mathrm{~d} s \geq C\left\|\left(u^{0}, u^{1}\right)\right\|_{V \times L^{2}(0,1)}^{2}, \quad \forall\left(u^{0}, u^{1}\right) \in V \times L^{2}(0,1) .
$$

The inequality above clearly contradicts assertion 3 in Proposition 5.8. So Assumption (5.38) is false. We end up in this way the proof of the first assertion of this theorem.

We pass now to the proof of the second assertion of this theorem. Let $\xi \in \mathcal{S}$. By Proposition 5.8, the solution $\phi$ of $(5.26-5.28)$ satisfies the inequality

$$
\int_{0}^{T}\left|\frac{\partial \phi}{\partial t}(\xi, t)\right|^{2} \mathrm{~d} t \geq K_{1}\left(\left\|u^{0}\right\|_{H^{1}(0,1)}^{2}+\left\|u^{1}\right\|_{H^{-1}(0,1)}^{2}\right), \forall\left(u^{0}, u^{1}\right) \in V \times L^{2}(0,1)
$$

where $K_{1}>0$ is a constant. The conclusion (5.24) follows now by simply using the Theorem 2.4 (with $\left.X_{1}=H^{1}(0,1), X_{2}=H^{-1}(0,1), Y_{1} \times Y_{2}=\left[H^{2}(0,1) \cap H^{4}(0,1) \cap H^{4}(\xi, 1)\right] \times H^{2}(0,1), \theta=\frac{2}{3}\right)$.

Let us now suppose that $\epsilon>0$ and that $\xi$ belongs to the set $B_{\epsilon}$, introduced in the proof of Proposition 5.4. From (5.34), it follows (5.25) by Theorem 2.4 (with $X_{1}=H^{1-\epsilon}(0,1), X_{2}=H^{-1-\epsilon}(0,1), Y_{1} \times Y_{2}$ $\left.=\left[H^{2}(0,1) \cap H^{4}(0,1) \cap H^{4}(\xi, 1)\right] \times H^{2}(0,1), \theta=\frac{2}{3+\epsilon}\right)$.

Remark 5.9. For different boundary condition there exist points $\xi \in(0,1)$ for which we have exponential decay (see Rebarber [20] and [1] for characterization of these points). 


\subsection{Third example: Dirichlet boundary stabilization of the wave equation in a disk}

Let $B_{R}=\left\{x \in \mathbb{R}^{2},\|x\|_{e}<R\right\}$ with boundary $\partial B_{R}=\bar{\Gamma}_{0} \cup \bar{\Gamma}_{1}$, where $\Gamma_{0}, \Gamma_{1}$ are disjoint parts of the boundary relatively open in $\partial B_{R}, \operatorname{int}\left(\Gamma_{0}\right) \neq \emptyset$. Above $\|.\|_{e}$ denotes the Euclidean norm in $\mathbb{R}^{2}$. We consider the wave equation:

$$
\begin{gathered}
\frac{\partial^{2} u}{\partial t^{2}}-\Delta u=0, B_{R} \times(0,+\infty), \\
u=\frac{\partial}{\partial \nu}\left(G u^{\prime}\right), \Gamma_{0} \times(0,+\infty), \\
u=0, \Gamma_{1} \times(0,+\infty), \\
u(x, 0)=u^{0}(x), \frac{\partial u}{\partial t}(x, 0)=u^{1}(x), B_{R},
\end{gathered}
$$

where $\nu$ is the unit normal vector of $\partial B_{R}$ pointing towards the exterior of $B_{R}$ and $G=(-\Delta)^{-1}: H^{-1}\left(B_{R}\right)$ $\rightarrow H_{0}^{1}\left(B_{R}\right)$.

Denote: $A=-\Delta, \mathcal{D}(A)=H_{0}^{1}\left(B_{R}\right)$. We will also denote by $A$ the extension of this operator $A: L^{2}\left(B_{R}\right)$ $\rightarrow\left(H^{2}\left(B_{R}\right) \cap H_{0}^{1}\left(B_{R}\right)\right)^{\prime}$. Notice that $\left(H^{2}\left(B_{R}\right) \cap H_{0}^{1}\left(B_{R}\right)\right)^{\prime}$ is the dual of $\mathcal{D}\left(A^{\frac{1}{2}}\right)$ with respect to the pivot space $H^{-1}\left(B_{R}\right)$. Moreover define

$$
B \in \mathcal{L}\left(L^{2}\left(\Gamma_{0}\right),\left(H^{2}\left(B_{R}\right) \cap H_{0}^{1}\left(B_{R}\right)\right)^{\prime}\right)
$$

by $B v=A D v, \forall v \in L^{2}\left(\Gamma_{0}\right)$, where $D \in \mathcal{L}\left(L^{2}\left(\Gamma_{0}\right), L^{2}\left(B_{R}\right)\right)$ is the Dirichlet map i.e., $D f=g$ if and only if

$$
\left\{\begin{array}{l}
\Delta g=0, B_{R} \\
g=f, \Gamma_{0}, g=0, \Gamma_{1}
\end{array}\right.
$$

Problem (5.39-5.42) can be rewritten in $V \times X=L^{2}\left(B_{R}\right) \times H^{-1}\left(B_{R}\right)$ as follows

$$
\left\{\begin{array}{c}
u^{\prime \prime}+A u+B B^{*} u^{\prime}=0 \\
u(0)=u^{0}, u^{\prime}(0)=u^{1}
\end{array}\right.
$$

Then, according to Proposition 2.1 the problem (5.39-5.42) is well-posed in $L^{2}\left(B_{R}\right) \times H^{-1}\left(B_{R}\right)$, i.e., for all $\left(u^{0}, u^{1}\right) \in L^{2}\left(B_{R}\right) \times H^{-1}\left(B_{R}\right)$, equations (5.39-5.42) admit a unique solution

$$
u \in C\left([0, \infty) ; L^{2}\left(B_{R}\right)\right) \cap C^{1}\left([0, \infty) ; H^{-1}\left(B_{R}\right)\right) .
$$

Moreover $u(x, t)$ satisfies the following energy estimate

$$
\left\|\left(u^{0}, u^{1}\right)\right\|_{L^{2}\left(B_{R}\right) \times H^{-1}\left(B_{R}\right)}^{2}-\left\|\left(u(t), \frac{\partial u}{\partial t}(t)\right)\right\|_{L^{2}\left(B_{R}\right) \times H^{-1}\left(B_{R}\right)}^{2}=2 \int_{0}^{t} \int_{\Gamma_{0}}\left|\frac{\partial\left[G\left(\frac{\partial u}{\partial t}\right)\right]}{\partial \nu}(x, s)\right|^{2} \mathrm{~d} \Gamma_{0} \mathrm{~d} s .
$$

Let $\phi$ be the solution of the following problem

$$
\frac{\partial^{2} \phi}{\partial t^{2}}-\Delta \phi=0, B_{R} \times(0, \infty)
$$




$$
\begin{gathered}
\phi=0, \partial B_{R} \times(0, \infty), \\
\phi(x, 0)=\phi^{0}(x), \frac{\partial \phi}{\partial t}(x, 0)=\phi^{1}(x), B_{R} .
\end{gathered}
$$

Denote

$$
\mathcal{A}_{d}=\left(\begin{array}{cc}
0 & I \\
\Delta & 0
\end{array}\right)
$$

with

$$
\mathcal{D}\left(\mathcal{A}_{d}\right)=\left\{(u, v) \in L^{2}\left(B_{R}\right) \times H^{-1}\left(B_{R}\right) /(v, \Delta u) \in L^{2}\left(B_{R}\right) \times H^{-1}\left(B_{R}\right), u_{\mid \Gamma_{0}}=\frac{\partial[G v]}{\partial \nu}, u_{\mid \Gamma_{1}}=0\right\}
$$

If $(u, v) \in \mathcal{D}\left(\mathcal{A}_{d}\right)$ we denote

$$
\|(u, v)\|_{\mathcal{D}\left(\mathcal{A}_{d}\right)}^{2}=\|(u, v)\|_{L^{2}\left(B_{R}\right) \times H^{-1}\left(B_{R}\right)}^{2}+\|(v, \Delta u)\|_{L^{2}\left(B_{R}\right) \times H^{-1}\left(B_{R}\right)}^{2} .
$$

The following holds.

Theorem 5.10. Suppose that there exists $T_{0}>0$ such that the solution $\phi$ of $(5.44-5.46)$ satisfy for all $T>T_{0}$

$$
\int_{0}^{T} \int_{\Gamma_{0}}\left|\frac{\partial \phi}{\partial \nu}\right|^{2} \mathrm{~d} \Gamma_{0} \mathrm{~d} t \geq C\left\|\left(\phi^{0}, \phi^{1}\right)\right\|_{H_{0}^{1}\left(B_{R}\right) \times L^{2}\left(B_{R}\right)}^{2}
$$

where $C$ is a positive constant which depends only on $T_{0}$.

Then all finite energy solutions $\left(u(t), u^{\prime}(t)\right)$ of (5.39-5.42) decay exponentially to zero in $L^{2}\left(B_{R}\right) \times H^{-1}\left(B_{R}\right)$.

Similar results were proved in a more general geometry and by different methods (see for instance Bardos et al. [4] and references therein).

Remark 5.11. Necessary and sufficient condition such that assumption (5.47) is satisfied is given in Bardos et al. [5].

Theorem 5.12. For all $\left(u^{0}, u^{1}\right) \in \mathcal{D}\left(\mathcal{A}_{d}\right)$ there exists a constant $K>0$ such that

$$
\left\|\left(u(t), \frac{\partial u}{\partial t}(t)\right)\right\|_{L^{2}\left(B_{R}\right) \times H^{-1}\left(B_{R}\right)}^{2} \leq \frac{K\left\|\left(u^{0}, u^{1}\right)\right\|_{\mathcal{D}\left(\mathcal{A}_{d}\right)}^{2}}{\ln (1+t)}, \forall t>0 .
$$

\section{Technicals results}

To prove Theorem 5.10 and Theorem 5.12 we need the following technicals results.

Let $\beta>0$ be a fixed real number and $C_{\beta}=\{\lambda \in \mathbb{C} \mid \operatorname{Re} \lambda=\beta\}$. Denote by $J_{l}$ the Bessel functions of integer order of first kind:

$$
\left\{\begin{array}{l}
\frac{1}{r} \frac{\mathrm{d}}{\mathrm{d} r}\left(r \frac{\mathrm{d} J_{l}}{\mathrm{~d} r}(r)\right)+\left(1-\frac{l^{2}}{r^{2}}\right) J_{l}(r)=0, \text { for } r>0 \\
J_{l} \text { is regular in } r=0
\end{array}\right.
$$


Proposition 5.13. There exists a constant $c_{\beta}>0$ (depends only on $\beta$ and $R$ ), such that

$$
\sup _{\lambda \in C_{\beta}} \frac{1}{|\lambda|}\left|i \lambda \frac{J_{l}^{\prime}(i \lambda R)}{J_{l}(i \lambda R)}-\frac{l}{R}\right| \leq c_{\beta} .
$$

Proof. The above estimate follows from the uniform asymptotic expansions of $J_{l}(z)$ (for $|l|$ and $|z|$ big enough). The reader is refered to Olver and Watson for the derivation and the proof of these asymptotic expansions. According to Olver and Watson, there are three regions for the asymptotic expansions of $J_{l}(z):|z| \gg|l|,|l| \ll|z|$ and the transition region where $|z| \sim|l|$. Making use of the formulas given in Olver [18] and Watson [27] (pp. 231 and 249), in each region we compute the asymptotic expansion of $\frac{J_{l}^{\prime}(z)}{J_{l}(z)}$. The following holds.

For $|l| \gg|\operatorname{Im} \lambda|$,

$$
\left|i \lambda \frac{J_{l}^{\prime}(i \lambda R)}{J_{l}(i \lambda R)}-\frac{l}{R}\right| \leq C,
$$

where $C$ is independant in $l$ and in $\operatorname{Im} \lambda$.

For $|l| \sim|\operatorname{Im} \lambda|$

$$
i \lambda \frac{J_{l}^{\prime}(i \lambda R)}{J_{l}(i \lambda R)} \sim \lambda^{\frac{2}{3}}
$$

For $|l| \ll|\operatorname{Im} \lambda|$,

$$
\left|i \lambda \frac{J_{l}^{\prime}(i \lambda R)}{J_{l}(i \lambda R)}-\frac{l}{R}\right| \leq C|\lambda|,
$$

where $C$ is independent in $l$ and $\operatorname{Im} \lambda$.

The estimates above end the proof.

Proposition 5.14. For all $\left(\phi^{0}, \phi^{1}\right) \in L^{2}\left(B_{R}\right) \times H^{-1}\left(B_{R}\right)$ and for all $\Gamma_{0} \subset \partial B_{R}$, int $\left(\Gamma_{0}\right) \neq \emptyset$, there exist $T, C_{1}>0$ such that

$$
\int_{0}^{T} \int_{\Gamma_{0}}\left|\frac{\partial}{\partial \nu}\left[G\left(\frac{\partial \phi}{\partial t}\right)\right]\right|^{2} \mathrm{~d} \Gamma_{0} \mathrm{~d} t \geq\left\|\phi^{0}, \phi^{1}\right\|_{L^{2}\left(B_{R}\right) \times H^{-1}\left(B_{R}\right)}^{2} \exp \left(-2 C_{1}^{2} \frac{\left\|\left(\phi^{0}, \phi^{1}\right)\right\|_{L^{2}\left(B_{R}\right) \times H^{-1}\left(B_{R}\right)}^{2}}{\left\|\left(\phi^{0}, \phi^{1}\right)\right\|_{H^{-1}\left(B_{R}\right) \times H^{-2}\left(B_{R}\right)}^{2}}\right)
$$

Proof. Let $\psi=G\left(\frac{\partial \phi}{\partial t}\right)$, then $\psi$ satisfy $(5.44,5.45)$ with $\left(\psi^{0}=G\left(\phi^{1}\right), \psi^{1}=-\phi_{0}\right) \in H_{0}^{1}\left(B_{R}\right) \times L^{2}\left(B_{R}\right)$. Thus, according to Theorem 1 in Robbiano [21], for all $\Gamma_{0} \subset \partial B_{R}$, there exist $T, C_{1}>0$ such that

$$
\left\|\left(G\left(\phi_{1}\right),-\phi_{0}\right)\right\|_{L^{2}\left(B_{R}\right) \times H^{-1}\left(B_{R}\right)} \leq \frac{C_{1}\left\|\left(G\left(\phi_{1}\right),-\phi_{0}\right)\right\|_{H_{0}^{1}\left(B_{R}\right) \times L^{2}\left(B_{R}\right)}}{\left[\ln \left(2+\frac{\left\|\left(G\left(\phi_{1}\right),-\phi_{0}\right)\right\|_{H_{0}^{1}\left(B_{R}\right) \times L^{2}\left(B_{R}\right)}}{\left(\int_{0}^{T} \int_{\Gamma_{0}}\left|\frac{\partial}{\partial \nu}\left[G\left(\frac{\partial \phi}{\partial t}\right)\right]\right|^{2} \mathrm{~d} \Gamma_{0} \mathrm{~d} t\right)^{\frac{1}{2}}}\right)\right]^{\frac{1}{2}}}
$$


Which obviously implies,

$$
\int_{0}^{T} \int_{\Gamma_{0}}\left|\frac{\partial}{\partial \nu}\left[G\left(\frac{\partial \phi}{\partial t}\right)\right]\right|^{2} \mathrm{~d} \Gamma_{0} \mathrm{~d} t \geq\left\|\phi^{0}, \phi^{1}\right\|_{L^{2}\left(B_{R}\right) \times H^{-1}\left(B_{R}\right)}^{2} \exp \left(-2 C_{1}^{2} \frac{\left\|\left(\phi^{0}, \phi^{1}\right)\right\|_{L^{2}\left(B_{R}\right) \times H^{-1}\left(B_{R}\right)}^{2}}{\left\|\left(\phi^{0}, \phi^{1}\right)\right\|_{H^{-1}\left(B_{R}\right) \times H^{-2}\left(B_{R}\right)}^{2}}\right) .
$$

Proof of Theorem 5.10. By Theorem 2.2 it suffices to verify $(\mathrm{H})$.

Notice first that

$$
B^{*} v=\frac{\partial[G(v)]}{\partial \nu}, \forall v \in L^{2}\left(B_{R}\right)
$$

The function $H(\lambda) \in \mathcal{L}\left(L^{2}\left(\Gamma_{0}\right)\right)$ associated to the problem (5.39-5.42) has an explicit representation in terms of Bessell functions. The function $w=\left(\lambda^{2}-\Delta\right)^{-1} B g$ satisfies

$$
\left\{\begin{array}{c}
\lambda^{2} w-\Delta w=0, B_{R} \\
w=g, \Gamma_{0}, w=0, \Gamma_{1}
\end{array}\right.
$$

where $g \in L^{2}\left(\Gamma_{0}\right)$.

If we consider the Fourier expansion

$$
f=\left\{\begin{array}{c}
g, \Gamma_{0}, \\
0, \Gamma_{1},
\end{array}=\sum_{l \in \mathbb{Z}} f_{l} \mathrm{e}^{i l \theta} \in L^{2}\left(\partial B_{R}\right),\right.
$$

a simple calculation using (5.49) shows

$$
w(x)=w(r, \theta)=\sum_{l \in \mathbb{Z}} \frac{J_{l}(i \lambda r)}{J_{l}(i \lambda R)} f_{l} \mathrm{e}^{i l \theta} .
$$

Thus,

$$
G(w)(x)=G(w)(r, \theta)=\frac{1}{\lambda^{2}} \sum_{l \in \mathbb{Z}}\left(\frac{J_{l}(i \lambda r)}{J_{l}(i \lambda R)}-\left(\frac{r}{R}\right)^{l}\right) f_{l} \mathrm{e}^{i l \theta} .
$$

Which obviously implies that

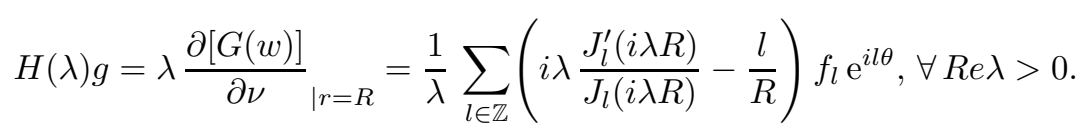

By Proposition 5.13 we obtain

$$
\|H(\lambda) g\|_{L^{2}\left(\Gamma_{0}\right)}^{2}=\frac{1}{|\lambda|^{2}} \sum_{l \in \mathbb{Z}}\left|i \lambda \frac{J_{l}^{\prime}(i \lambda R)}{J_{l}(i \lambda R)}-\frac{l}{R}\right|^{2}\left|f_{l}\right|^{2} \leq c_{\beta}^{2}\|f\|_{L^{2}\left(\partial B_{R}\right)}^{2}=c_{\beta}^{2}\|g\|_{L^{2}\left(\Gamma_{0}\right)}^{2},
$$

where the positive constant $c_{\beta}$ depends only on $\beta$ and $R$. From this estimate we conclude that the function $H(\lambda)$ is bounded on $C_{\beta}$, i.e. ( $\left.\mathrm{H}\right)$ holds true.

Thus, making use of Theorem 2.2, we obtain the exponential stability of all finite energy solutions of $(5.39-5.42)$. 
Proof of Theorem 5.12. According to Proposition 5.14, for all $\left(u^{0}, u^{1}\right) \in L^{2}\left(B_{R}\right) \times H^{-1}\left(B_{R}\right)$ and for all $\Gamma_{0} \subset \partial B_{R}$, there exist $T>0$ and $C_{1}>0$ such that

$$
\int_{0}^{T}\left\|\frac{\partial\left[G\left(\frac{\partial \phi}{\partial t}\right)\right]}{\partial \nu}\right\|_{L^{2}\left(\Gamma_{0}\right)}^{2} \mathrm{~d} t \geq\left\|\left(u^{0}, u^{1}\right)\right\|_{L^{2}\left(B_{R}\right) \times H^{-1}\left(B_{R}\right)}^{2} \exp \left(-2 C_{1}^{2} \frac{\left\|\left(u^{0}, u^{1}\right)\right\|_{L^{2}\left(B_{R}\right) \times H^{-1}\left(B_{R}\right)}^{2}}{\left\|\left(u^{0}, u^{1}\right)\right\|_{H^{-1}\left(B_{R}\right) \times H^{-2}\left(B_{R}\right)}^{2}}\right) .
$$

Since $(\mathrm{H})$ is satisfied (see the proof of Th. 5.10), then Lemma 4.1 implies that the solution $u(x, t)$ of $(5.39-5.42)$ satisfies the following inequality

$$
\begin{gathered}
\int_{0}^{T}\left\|\frac{\partial\left[G\left(\frac{\partial u}{\partial t}\right)\right]}{\partial \nu}\right\|_{L^{2}\left(\Gamma_{0}\right)}^{2} \mathrm{~d} t \geq C\left\|\left(u^{0}, u^{1}\right)\right\|_{L^{2}\left(B_{R}\right) \times H^{-1}\left(B_{R}\right)}^{2} \exp \left(-2 C_{1}^{2} \frac{\left\|\left(u^{0}, u^{1}\right)\right\|_{L^{2}\left(B_{R}\right) \times H^{-1}\left(B_{R}\right)}^{2}}{\left\|\left(u^{0}, u^{1}\right)\right\|_{H^{-1}\left(B_{R}\right) \times H^{-2}\left(B_{R}\right)}^{2}}\right) \\
\forall\left(u^{0}, u^{1}\right) \in L^{2}\left(B_{R}\right) \times H^{-1}\left(B_{R}\right)
\end{gathered}
$$

Estimate (5.48) follow from Theorem 2.4 (with $X_{1}=H^{-1}\left(B_{R}\right), X_{2}=H^{-2}\left(B_{R}\right), Y_{1} \times Y_{2}=H^{1}\left(B_{R}\right)$ $\left.\times L^{2}\left(B_{R}\right), \mathcal{G}(x)=\exp \left(-\frac{2 C_{1}^{2}}{x}\right), \forall x>0, \theta=\frac{1}{2}\right)$.

The authors thank the referee for his helpful suggestions and comments.

\section{REFERENCES}

[1] K. Ammari and M. Tucsnak, Stabilization of Bernoulli-Euler beams by means of a pointwise feedback force. SIAM. J. Control Optim. 39 (2000) 1160-1181.

[2] K. Ammari, A. Henrot and M. Tucsnak, Optimal location of the actuator for the pointwise stabilization of a string. $C . R$. Acad. Sci. Paris Sér. I Math. 330 (2000) 275-280.

[3] A. Bamberger, J. Rauch and M. Taylor, A model for harmonics on stringed instruments. Arch. Rational Mech. Anal. 79 (1982) 267-290.

[4] C. Bardos, L. Halpern, G. Lebeau, J. Rauch and E. Zuazua, Stabilisation de l'équation des ondes au moyen d'un feedback portant sur la condition aux limites de Dirichlet. Asymptot. Anal. 4 (1991) 285-291.

[5] C. Bardos, G. Lebeau and J. Rauch, Sharp sufficient conditions for the observation, control and stabilization of waves from the boundary. SIAM J. Control Optim. 30 (1992) 1024-1065.

[6] A. Bensoussan, G. Da Prato, M.C. Delfour and S.K. Mitter, Representation and control of infinite Dimensional Systems, Vol. I. Birkhauser (1992).

[7] J.W.S. Cassals, An introduction to Diophantine Approximation. Cambridge University Press, Cambridge (1966).

[8] G. Doetsch, Introduction to the theory and application of the Laplace transformation. Springer, Berlin (1974).

[9] A. Haraux, Une remarque sur la stabilisation de certains systèmes du deuxième ordre en temps. Portugal Math. 46 (1989) 245-258.

[10] A.E. Ingham, Some trigonometrical inequalities with applications in the theory of series. Math. Z. 41 (1936) 367-369.

[11] S. Jaffard, M. Tucsnak and E. Zuazua, Singular internal stabilization of the wave equation. J. Differential Equations 145 (1998) 184-215.

[12] V. Komornik, Rapid boundary stabilization of linear distributed systems. SIAM J. Control Optim. 35 (1997) 1591-1613.

[13] V. Komornik and E. Zuazua, A direct method for the boundary stabilization of the wave equation. J. Math. Pures Appl. 69 (1990) 33-54.

[14] J. Lagnese, Boundary stabilization of thin plates. Philadelphia, SIAM Stud. Appl. Math. (1989).

[15] S. Lang, Introduction to diophantine approximations. Addison Wesley, New York (1966).

[16] J.L. Lions, Contrôlabilité exacte des systèmes distribués. Masson, Paris (1998).

[17] J.L. Lions and E. Magenes, Problèmes aux limites non homogènes et applications, Vol. 1. Dunod, Paris (1968).

[18] F.W.J. Olver, Asymptotic and Special Functions. Academic Press, New York. 
[19] A. Pazy, Semigroups of linear operators and applications to partial differential equations. Springer, New York (1983).

[20] R. Rebarber, Exponential stability of beams with dissipative joints: A frequency approach. SIAM J. Control Optim. 33 (1995) $1-28$.

[21] L. Robbiano, Fonction de coût et contrôle des solutions des équations hyperboliques. Asymptot. Anal. 10 (1995) 95-115.

$[22]$ D.L. Russell, Decay rates for weakly damped systems in Hilbert space obtained with control theoretic methods. J. Differential Equations 19 (1975) 344-370.

[23] D.L. Russell, Controllability and stabilizability theory for linear partial differential equations: Recent and open questions. SIAM Rev. 20 (1978) 639-739.

[24] H. Triebel, Interpolation theory, function spaces, differential operators. North Holland, Amsterdam (1978).

[25] M. Tucsnak, Regularity and exact controllability for a beam with piezoelectric actuator. SIAM J. Control Optim. 34 (1996) 922-930.

[26] M. Tucsnak and G. Weiss, How to get a conservative well posed linear system out of thin air. Preprint.

[27] G.N. Watson, A treatise on the theory of Bessel functions. Cambridge University Press.

[28] G. Weiss, Regular linear systems with feedback. Math. Control Signals Systems 7 (1994) 23-57. 